\title{
Table S7
}

A) Association results of nominally significant SNPs with Type 2 Diabetes in the DIAGRAM+ Consortium.

\begin{tabular}{|c|c|c|c|c|c|c|}
\hline SNP & Chr/position & Closest Gene & EA/NEA & OR $(95 \%$ CI $)$ & $\mathbf{P}$ & $\mathbf{N}$ \\
\hline rs 1648707 & $3 / 188034413$ & $A D I P O Q$ & $\mathrm{C} / \mathrm{A}$ & $1.045(1.002,1.089)$ & 0.039 & 22,570 \\
\hline rs6444168 & $3 / 188035559$ & $A D I P O Q$ & $\mathrm{G} / \mathrm{A}$ & $1.069(1.005,1.136)$ & 0.034 & 21,520 \\
\hline rs 266729 & $3 / 188042176$ & $A D I P O Q$ & $\mathrm{G} / \mathrm{C}$ & $1.061(1.014,1.111)$ & 0.011 & 22,570 \\
\hline rs182052 & $3 / 188043484$ & $A D I P O Q$ & $\mathrm{~A} / \mathrm{G}$ & $1.043(1,1.087)$ & 0.048 & 22,570 \\
\hline rs7649121 & $3 / 188051487$ & $A D I P O Q$ & $\mathrm{~T} / \mathrm{A}$ & $1.122(1.041,1.209)$ & 0.003 & 18,352 \\
\hline rs 863750 & $12 / 123030324$ & ZNF664 & $\mathrm{T} / \mathrm{C}$ & $1.051(1.008,1.096)$ & 0.020 & 22,570 \\
\hline rs 10773049 & $12 / 123031511$ & ZNF664 & $\mathrm{T} / \mathrm{C}$ & $1.048(1.005,1.093)$ & 0.030 & 22,570 \\
\hline rs825453 & $12 / 123033638$ & ZNF664 & $\mathrm{T} / \mathrm{A}$ & $1.049(1.006,1.094)$ & 0.026 & 22,570 \\
\hline rs2927322 & $16 / 80072006$ & CMIP & $\mathrm{A} / \mathrm{G}$ & $1.065(1.017,1.114)$ & 0.007 & 22,570 \\
\hline rs 12443634 & $16 / 80081775$ & CMIP & $\mathrm{A} / \mathrm{C}$ & $1.049(1.001,1.098)$ & 0.045 & 21,198 \\
\hline rs2925979 & $16 / 80092291$ & CMIP & $\mathrm{T} / \mathrm{C}$ & $1.066(1.02,1.115)$ & 0.005 & 21,198 \\
\hline rs2966093 & $16 / 80096121$ & CMIP & $\mathrm{G} / \mathrm{A}$ & $1.051(1.005,1.099)$ & 0.030 & 21,198 \\
\hline rs2966094 & $16 / 80096138$ & CMIP & $\mathrm{A} / \mathrm{C}$ & $1.055(1.009,1.103)$ & 0.019 & 21,198 \\
\hline
\end{tabular}

EA: Effect Allele

NEA: Non-Effect Allele 
Table S7

B) Association results of nominally significant SNPs with diabetes-related traits in the MAGIC Consortium.

\begin{tabular}{|c|c|c|c|c|c|c|c|c|}
\hline SNP & Chr/position & Closest Gene & EA/NEA & Beta & SE & $\mathbf{P}$ & $\mathbf{N}$ & associated traits \\
\hline rs1108842 & $3 / 52695120$ & GNL3 & $\mathrm{A} / \mathrm{C}$ & 0.009 & 0.004 & 0.027 & 37,209 & FI \\
\hline rs2710323 & $3 / 52790945$ & ITIH1 & $\mathrm{T} / \mathrm{C}$ & 0.009 & 0.004 & 0.019 & 37,197 & FI \\
\hline rs 3617 & $3 / 52808845$ & ITIH3 & $\mathrm{A} / \mathrm{C}$ & -0.008 & 0.004 & 0.031 & 37,150 & FI \\
\hline rs4481150 & $3 / 52812833$ & ITIH3 & $\mathrm{T} / \mathrm{C}$ & 0.008 & 0.004 & 0.042 & 37,801 & FI \\
\hline rs 2535627 & $3 / 52820145$ & ITIH4 & $\mathrm{T} / \mathrm{C}$ & 0.009 & 0.004 & 0.020 & 36,776 & FI \\
\hline rs 2535627 & $3 / 52820145$ & ITIH4 & $\mathrm{T} / \mathrm{C}$ & 0.008 & 0.004 & 0.041 & 35,477 & $\mathrm{HbA} 1 \mathrm{C}$ \\
\hline rs 2535627 & $3 / 52820145$ & ITIH4 & $\mathrm{T} / \mathrm{C}$ & 0.008 & 0.004 & 0.041 & 35,477 & Homa-IR \\
\hline rs2071044 & $3 / 52822641$ & ITIH4 & $\mathrm{T} / \mathrm{C}$ & -0.008 & 0.004 & 0.037 & 37,802 & FI \\
\hline rs7617025 & $3 / 151538648$ & $T S C 22 D 2$ & $\mathrm{~A} / \mathrm{G}$ & 0.013 & 0.006 & 0.040 & 46,186 & FG \\
\hline rs1972703 & $3 / 187946037$ & $K N G 1$ & $\mathrm{~A} / \mathrm{G}$ & 0.061 & 0.027 & 0.024 & 15,234 & 2hr-Glu \\
\hline rs266749 & $3 / 187954613$ & $K N G 1$ & $\mathrm{~T} / \mathrm{C}$ & 0.009 & 0.004 & 0.037 & 44,713 & FG \\
\hline rs266742 & $3 / 187958539$ & $K N G 1$ & $\mathrm{~T} / \mathrm{G}$ & -0.008 & 0.004 & 0.048 & 45,972 & FG \\
\hline rs1501299 & $3 / 188053817$ & $A D I P O Q$ & $\mathrm{~T} / \mathrm{G}$ & -0.016 & 0.007 & 0.022 & 14,398 & FI \\
\hline rs1501299 & $3 / 188053817$ & $A D I P O Q$ & $\mathrm{~T} / \mathrm{G}$ & -0.015 & 0.008 & 0.048 & 13,363 & $\mathrm{HbA} 1 \mathrm{C}$ \\
\hline rs1501299 & $3 / 188053817$ & $A D I P O Q$ & $\mathrm{~T} / \mathrm{G}$ & -0.015 & 0.008 & 0.048 & 13,363 & Homa-IR \\
\hline rs 12812995 & $12 / 20369141$ & $P D E 3 A$ & $\mathrm{C} / \mathrm{G}$ & -0.042 & 0.020 & 0.035 & 15,234 & 2hr-Glu \\
\hline rs1444636 & $12 / 20384150$ & $P D E 3 A$ & $\mathrm{C} / \mathrm{G}$ & -0.046 & 0.020 & 0.018 & 15,234 & 2hr-Glu \\
\hline rs 10770643 & $12 / 20385101$ & $P D E 3 A$ & $\mathrm{~A} / \mathrm{G}$ & 0.047 & 0.020 & 0.016 & 15,234 & 2hr-Glu \\
\hline rs7303397 & $12 / 20385638$ & PDE3A & $\mathrm{A} / \mathrm{G}$ & 0.049 & 0.019 & 0.012 & 15,234 & 2hr-Glu \\
\hline rs7955516 & $12 / 20389303$ & $P D E 3 A$ & $\mathrm{~A} / \mathrm{C}$ & 0.045 & 0.020 & 0.021 & 15,234 & 2hr-Glu \\
\hline rs2120757 & $12 / 20393267$ & $P D E 3 A$ & $\mathrm{~T} / \mathrm{C}$ & 0.045 & 0.019 & 0.021 & 15,234 & 2hr-Glu \\
\hline rs2009084 & $12 / 20402460$ & $P D E 3 A$ & $\mathrm{~T} / \mathrm{C}$ & 0.047 & 0.020 & 0.016 & 15,234 & 2hr-Glu \\
\hline rs 11057405 & $12 / 121347850$ & CLIP1 & $\mathrm{A} / \mathrm{G}$ & 0.017 & 0.008 & 0.033 & 32,319 & FI \\
\hline rs 2454722 & $12 / 121737171$ & GPR109A & $\mathrm{A} / \mathrm{G}$ & 0.012 & 0.005 & 0.024 & 35,909 & $\mathrm{HbA} 1 \mathrm{C}$ \\
\hline rs 2454722 & $12 / 121737171$ & GPR109A & $\mathrm{A} / \mathrm{G}$ & 0.012 & 0.005 & 0.024 & 35,909 & Homa-IR \\
\hline rs 2454722 & $12 / 121737171$ & GPR109A & $\mathrm{A} / \mathrm{G}$ & 0.011 & 0.005 & 0.031 & 37,208 & FI \\
\hline rs601339 & $12 / 121740696$ & GPR109A & $\mathrm{A} / \mathrm{G}$ & 0.012 & 0.005 & 0.028 & 37,032 & $\mathrm{HbA1C}$ \\
\hline rs601339 & $12 / 121740696$ & GPR109A & $\mathrm{A} / \mathrm{G}$ & 0.012 & 0.005 & 0.028 & 37,032 & Homa-IR \\
\hline rs601339 & $12 / 121740696$ & GPR109A & $\mathrm{A} / \mathrm{G}$ & 0.011 & 0.005 & 0.036 & 38,234 & FI \\
\hline rs4759361 & $12 / 121744233$ & GPR109A & $\mathrm{A} / \mathrm{T}$ & -0.012 & 0.005 & 0.026 & 37,032 & $\mathrm{HbA} 1 \mathrm{C}$ \\
\hline rs4759361 & $12 / 121744233$ & GPR109A & $\mathrm{A} / \mathrm{T}$ & -0.012 & 0.005 & 0.026 & 37,032 & Homa-IR \\
\hline rs4759361 & $12 / 121744233$ & GPR109A & $\mathrm{A} / \mathrm{T}$ & -0.011 & 0.005 & 0.034 & 38,234 & FI \\
\hline rs509548 & $12 / 121747808$ & GPR109A & $\mathrm{A} / \mathrm{T}$ & -0.012 & 0.005 & 0.028 & 37,032 & $\mathrm{HbA1C}$ \\
\hline rs509548 & $12 / 121747808$ & GPR109A & $\mathrm{A} / \mathrm{T}$ & -0.012 & 0.005 & 0.028 & 37,032 & Homa-IR \\
\hline rs509548 & $12 / 121747808$ & GPR109A & $\mathrm{A} / \mathrm{T}$ & -0.011 & 0.005 & 0.036 & 38,234 & FI \\
\hline rs6488898 & $12 / 122769785$ & ATP6V0A2 & $\mathrm{A} / \mathrm{G}$ & -0.073 & 0.037 & 0.049 & 15,234 & 2hr-Glu \\
\hline rs 11057354 & $12 / 122833591$ & DNAH10 & $\mathrm{A} / \mathrm{G}$ & 0.057 & 0.029 & 0.048 & 15,234 & 2hr-Glu \\
\hline rs 11057401 & $12 / 122993259$ & CCDC92 & $\mathrm{A} / \mathrm{T}$ & -0.008 & 0.004 & 0.044 & 46,170 & FG \\
\hline rs 2178663 & $12 / 122999858$ & CCDC92 & $\mathrm{T} / \mathrm{C}$ & -0.008 & 0.004 & 0.049 & 46,185 & FG \\
\hline rs3867146 & $12 / 123003958$ & CCDC92 & $\mathrm{A} / \mathrm{G}$ & 0.016 & 0.006 & 0.014 & 34,204 & FI \\
\hline rs3867146 & $12 / 123003958$ & CCDC92 & $\mathrm{A} / \mathrm{G}$ & 0.014 & 0.007 & 0.036 & 32,954 & $\mathrm{HbA1C}$ \\
\hline rs3867146 & $12 / 123003958$ & $C C D C 92$ & $\mathrm{~A} / \mathrm{G}$ & 0.014 & 0.007 & 0.036 & 32,954 & Homa-IR \\
\hline rs3867146 & $12 / 123003958$ & CCDC92 & $\mathrm{A} / \mathrm{G}$ & 0.060 & 0.029 & 0.040 & 13,563 & 2hr-Glu \\
\hline rs11831913 & $12 / 123012851$ & $C C D C 92$ & $\mathrm{~T} / \mathrm{C}$ & 0.008 & 0.004 & 0.045 & 46,186 & FG \\
\hline rs4765148 & $12 / 123044590$ & ZNF664 & $\mathrm{T} / \mathrm{G}$ & -0.008 & 0.004 & 0.044 & 45,951 & FG \\
\hline rs2130382 & $12 / 123050596$ & ZNF664 & $\mathrm{C} / \mathrm{G}$ & 0.008 & 0.004 & 0.041 & 46,184 & FG \\
\hline rs 7307053 & $12 / 123060493$ & ZNF664 & $\mathrm{T} / \mathrm{C}$ & -0.008 & 0.004 & 0.040 & 46,186 & FG \\
\hline rs 12824567 & $12 / 123061156$ & ZNF664 & $\mathrm{C} / \mathrm{G}$ & -0.008 & 0.004 & 0.039 & 46,186 & FG \\
\hline
\end{tabular}




\begin{tabular}{|c|c|c|c|c|c|c|c|c|}
\hline rs 863750 & $12 / 123071397$ & ZNF664 & $\mathrm{T} / \mathrm{C}$ & 0.009 & 0.004 & 0.028 & 37,187 & FI \\
\hline rs 10773049 & $12 / 123072584$ & ZNF664 & $\mathrm{T} / \mathrm{C}$ & 0.009 & 0.004 & 0.029 & 38,231 & FI \\
\hline rs825453 & $12 / 123074711$ & ZNF664 & $\mathrm{A} / \mathrm{T}$ & -0.009 & 0.004 & 0.031 & 37,801 & FI \\
\hline rs11057418 & $12 / 123074929$ & ZNF664 & $\mathrm{C} / \mathrm{G}$ & 0.059 & 0.024 & 0.012 & 15,234 & 2hr-Glu \\
\hline rs 11057418 & $12 / 123074929$ & ZNF664 & $\mathrm{C} / \mathrm{G}$ & 0.010 & 0.005 & 0.037 & 38,233 & FI \\
\hline rs 2927328 & $16 / 80067223$ & $C M I P$ & $\mathrm{~T} / \mathrm{C}$ & 0.044 & 0.020 & 0.026 & 15,234 & 2hr-Glu \\
\hline rs7193788 & $16 / 81213661$ & $\mathrm{CDH} 13$ & $\mathrm{~A} / \mathrm{G}$ & -0.015 & 0.005 & 0.005 & 35,910 & $\mathrm{HbA} 1 \mathrm{C}$ \\
\hline rs7193788 & $16 / 81213661$ & $\mathrm{CDH13}$ & $\mathrm{A} / \mathrm{G}$ & -0.015 & 0.005 & 0.005 & 35,910 & Homa-IR \\
\hline rs7193788 & $16 / 81213661$ & CDH13 & $\mathrm{A} / \mathrm{G}$ & -0.013 & 0.005 & 0.013 & 37,208 & FI \\
\hline rs7193788 & $16 / 81213661$ & $C D H 13$ & $\mathrm{~A} / \mathrm{G}$ & -0.010 & 0.004 & 0.024 & 35,444 & Homa-b \\
\hline rs 2163869 & $16 / 81223356$ & CDH13 & $\mathrm{A} / \mathrm{G}$ & 0.009 & 0.004 & 0.032 & 42,602 & FG \\
\hline rs889140 & $19 / 38580840$ & $P E P D$ & $\mathrm{~A} / \mathrm{G}$ & -0.009 & 0.004 & 0.031 & 37,210 & FI \\
\hline rs889140 & $19 / 38580840$ & PEPD & $\mathrm{A} / \mathrm{G}$ & -0.009 & 0.004 & 0.033 & 35,912 & $\mathrm{HbA} 1 \mathrm{C}$ \\
\hline rs889140 & $19 / 38580840$ & $P E P D$ & $\mathrm{~A} / \mathrm{G}$ & -0.009 & 0.004 & 0.033 & 35,912 & Homa-IR \\
\hline rs889139 & $19 / 38581209$ & $P E P D$ & $\mathrm{~A} / \mathrm{G}$ & 0.009 & 0.004 & 0.038 & 36,975 & $\mathrm{HbA1C}$ \\
\hline rs889139 & $19 / 38581209$ & $P E P D$ & $\mathrm{~A} / \mathrm{G}$ & 0.009 & 0.004 & 0.038 & 36,975 & Homa-IR \\
\hline rs889139 & $19 / 38581209$ & $P E P D$ & $\mathrm{~A} / \mathrm{G}$ & 0.008 & 0.004 & 0.039 & 38,176 & FI \\
\hline rs731839 & $19 / 38590905$ & $P E P D$ & $\mathrm{~A} / \mathrm{G}$ & -0.011 & 0.004 & 0.009 & 37,802 & FI \\
\hline rs 731839 & $19 / 38590905$ & $P E P D$ & $\mathrm{~A} / \mathrm{G}$ & -0.011 & 0.004 & 0.014 & 36,601 & $\mathrm{HbA1C}$ \\
\hline rs 731839 & $19 / 38590905$ & $P E P D$ & $\mathrm{~A} / \mathrm{G}$ & -0.011 & 0.004 & 0.014 & 36,601 & Homa-IR \\
\hline rs 4805885 & $19 / 38597963$ & $P E P D$ & $\mathrm{~T} / \mathrm{C}$ & 0.012 & 0.004 & 0.002 & 37,802 & FI \\
\hline rs 4805885 & $19 / 38597963$ & $P E P D$ & $\mathrm{~T} / \mathrm{C}$ & 0.012 & 0.004 & 0.003 & 36,601 & $\mathrm{HbA} 1 \mathrm{C}$ \\
\hline rs 4805885 & $19 / 38597963$ & $P E P D$ & $\mathrm{~T} / \mathrm{C}$ & 0.012 & 0.004 & 0.003 & 36,601 & Homa-IR \\
\hline rs8182584 & $19 / 38601550$ & $P E P D$ & $\mathrm{~T} / \mathrm{G}$ & 0.012 & 0.004 & 0.002 & 36,776 & FI \\
\hline rs 8182584 & $19 / 38601550$ & $P E P D$ & $\mathrm{~T} / \mathrm{G}$ & 0.012 & 0.004 & 0.004 & 35,478 & $\mathrm{HbA1C}$ \\
\hline rs8182584 & $19 / 38601550$ & $P E P D$ & $\mathrm{~T} / \mathrm{G}$ & 0.012 & 0.004 & 0.004 & 35,478 & Homa-IR \\
\hline rs 8182584 & $19 / 38601550$ & $P E P D$ & $\mathrm{~T} / \mathrm{G}$ & 0.007 & 0.004 & 0.041 & 35,012 & Homa-b \\
\hline
\end{tabular}

Fasting glucose and $2 \mathrm{~h}$ glucose in $\mathrm{mmol} / \mathrm{L}$; Insulin in $\mathrm{pmol} / \mathrm{L}$.

EA: Effect Allele

NEA: Non-Effect Allele 
Table S7

C) Association results of nominally significant SNPs with diabetes-related traits in the GIANT and Body fat GWAS consortia.

\begin{tabular}{|c|c|c|c|c|c|c|c|c|}
\hline SNP & Chr/position & Closest Gene & EA/NEA & Beta & SE & $\mathbf{P}$ & $\mathbf{N}$ & associated traits \\
\hline rs 13081028 & $3 / 52530356$ & STAB1 & $\mathbf{A} / \mathbf{G}$ & -0.021 & 0.005 & $1.26 \mathrm{E}-05$ & 77,167 & WHR \\
\hline rs9853056 & $3 / 52530997$ & $S T A B 1$ & $\mathbf{T} / \mathbf{C}$ & 0.021 & 0.005 & $1.28 \mathrm{E}-05$ & 77,167 & WHR \\
\hline rs4282054 & $3 / 52541105$ & NT5DC2 & $\mathbf{T} / \mathbf{C}$ & 0.021 & 0.005 & $1.73 E-05$ & 77,167 & WHR \\
\hline rs2590838 & $3 / 52597126$ & PBRM1 & $\mathbf{A} / \mathbf{G}$ & -0.020 & 0.005 & 3.06E-05 & 77,167 & WHR \\
\hline rs2276824 & $3 / 52612526$ & PBRM1 & $\mathrm{C} / \mathrm{G}$ & 0.019 & 0.005 & $2.80 \mathrm{E}-04$ & 77,167 & WHR \\
\hline rs9879090 & $3 / 52623305$ & PBRM1 & $\mathbf{T} / \mathbf{C}$ & 0.019 & 0.005 & 2.70E-04 & 77,167 & WHR \\
\hline rs13083798 & $3 / 52624788$ & PBRMI & $\mathbf{A} / \mathbf{G}$ & 0.020 & 0.005 & $1.95 \mathrm{E}-05$ & 77,167 & WHR \\
\hline rs1108842 & $3 / 52695120$ & GNL3 & $\mathbf{A} / \mathbf{C}$ & 0.021 & 0.005 & $1.68 \mathrm{E}-05$ & 77,167 & WHR \\
\hline rs11235 & 3/52720127 & NEK4 & $\mathbf{T} / \mathbf{C}$ & -0.019 & 0.005 & 3.10E-04 & 77,167 & WHR \\
\hline rs2710323 & $3 / 52790945$ & ITIHI & $\mathbf{T} / \mathbf{C}$ & 0.022 & 0.005 & $7.21 \mathrm{E}-06$ & 77,167 & WHR \\
\hline rs3617 & $3 / 52808845$ & ITIH3 & $\mathrm{A} / \mathrm{C}$ & -0.020 & 0.005 & 4.59E-05 & 77,167 & WHR \\
\hline rs3617 & $3 / 52808845$ & ІТІНЗ & $\mathrm{A} / \mathrm{C}$ & 0.010 & 0.005 & 2.99E-02 & 123,745 & BMI \\
\hline rs4481150 & $3 / 52812833$ & ITIH3 & $\mathbf{T} / \mathbf{C}$ & 0.019 & 0.005 & 1.00E-04 & 77,167 & WHR \\
\hline rs2535627 & $3 / 52820145$ & ITIH 4 & $\mathbf{T} / \mathbf{C}$ & 0.019 & 0.005 & 8.22E-05 & 77,167 & WHR \\
\hline rs2071044 & $3 / 52822641$ & ITIH4 & $\mathbf{T} / \mathbf{C}$ & -0.019 & 0.005 & $1.10 \mathrm{E}-04$ & 77,167 & WHR \\
\hline rs11927941 & $3 / 187915740$ & $K N G 1$ & $\mathrm{~A} / \mathrm{G}$ & 0.017 & 0.008 & $4.00 \mathrm{E}-02$ & 34,832 & fat $\%$ \\
\hline rs 3774291 & $3 / 187916165$ & $K N G 1$ & $\mathrm{~T} / \mathrm{C}$ & -0.017 & 0.008 & $4.20 \mathrm{E}-02$ & 34,832 & fat $\%$ \\
\hline rs3774292 & $3 / 187916268$ & $K N G 1$ & $\mathrm{~A} / \mathrm{T}$ & 0.016 & 0.008 & $3.70 \mathrm{E}-02$ & 36,608 & fat $\%$ \\
\hline rs10440056 & $3 / 187916516$ & $K N G 1$ & $\mathrm{~T} / \mathrm{C}$ & -0.016 & 0.008 & $3.70 \mathrm{E}-02$ & 36,608 & fat $\%$ \\
\hline rs3821815 & $3 / 187916956$ & $K N G 1$ & $\mathrm{~T} / \mathrm{C}$ & 0.016 & 0.008 & $3.70 \mathrm{E}-02$ & 36,619 & fat $\%$ \\
\hline rs1851665 & $3 / 187919092$ & $K N G 1$ & $\mathrm{~A} / \mathrm{G}$ & 0.016 & 0.008 & $3.80 \mathrm{E}-02$ & 36,610 & fat $\%$ \\
\hline rs1836860 & $3 / 187919298$ & $K N G 1$ & $\mathrm{~T} / \mathrm{C}$ & 0.016 & 0.008 & $4.10 \mathrm{E}-02$ & 36,611 & fat $\%$ \\
\hline rs1656925 & $3 / 187931817$ & $K N G 1$ & $\mathrm{~T} / \mathrm{C}$ & 0.015 & 0.007 & $4.40 \mathrm{E}-02$ & 36,625 & fat $\%$ \\
\hline rs1648700 & $3 / 187932610$ & $K N G 1$ & $\mathrm{~T} / \mathrm{C}$ & 0.015 & 0.007 & $4.20 \mathrm{E}-02$ & 36,613 & fat $\%$ \\
\hline rs1624569 & $3 / 187932763$ & $K N G 1$ & $\mathrm{~T} / \mathrm{C}$ & 0.015 & 0.007 & $4.00 \mathrm{E}-02$ & 36,601 & fat $\%$ \\
\hline rs710448 & $3 / 187935579$ & $K N G 1$ & $\mathrm{~A} / \mathrm{G}$ & 0.015 & 0.007 & $4.40 \mathrm{E}-02$ & 36,622 & fat $\%$ \\
\hline rs 822363 & $3 / 187935685$ & $K N G 1$ & $\mathrm{C} / \mathrm{G}$ & 0.015 & 0.008 & $4.30 \mathrm{E}-02$ & 36,622 & fat $\%$ \\
\hline rs2062632 & $3 / 187943875$ & $K N G 1$ & $\mathrm{~T} / \mathrm{C}$ & -0.013 & 0.006 & $3.90 \mathrm{E}-02$ & 77,167 & WHR \\
\hline rs1426810 & $3 / 187986129$ & EIF $4 A 2$ & $\mathrm{~A} / \mathrm{G}$ & 0.016 & 0.008 & $3.10 \mathrm{E}-02$ & 36,622 & fat $\%$ \\
\hline rs1354091 & $3 / 187988594$ & $E I F 4 A 2$ & $\mathrm{~T} / \mathrm{G}$ & -0.018 & 0.008 & $3.50 \mathrm{E}-02$ & 36,611 & fat $\%$ \\
\hline rs 2066500 & $3 / 187990616$ & RFC4 & $\mathrm{T} / \mathrm{C}$ & 0.018 & 0.008 & $3.40 \mathrm{E}-02$ & 36,607 & fat $\%$ \\
\hline rs3917117 & $3 / 187998538$ & RFC4 & $\mathrm{A} / \mathrm{G}$ & 0.018 & 0.009 & $3.50 \mathrm{E}-02$ & 36,597 & fat $\%$ \\
\hline rs3917110 & $3 / 188001346$ & RFC4 & $\mathrm{C} / \mathrm{G}$ & -0.017 & 0.008 & $4.20 \mathrm{E}-02$ & 36,604 & fat $\%$ \\
\hline rs3917109 & $3 / 188001500$ & RFC4 & $\mathrm{A} / \mathrm{C}$ & 0.028 & 0.012 & $1.70 \mathrm{E}-02$ & 18,028 & fat $\%$ \\
\hline rs16861184 & $3 / 188003171$ & RFC4 & $\mathrm{T} / \mathrm{C}$ & 0.017 & 0.008 & 4.10E-02 & 36,604 & fat $\%$ \\
\hline rs710450 & $3 / 188005327$ & $R F C 4$ & $\mathrm{~A} / \mathrm{C}$ & 0.016 & 0.007 & $3.10 \mathrm{E}-02$ & 77,167 & WHR \\
\hline rs2293243 & $3 / 188005431$ & $R F C 4$ & $\mathrm{~A} / \mathrm{T}$ & -0.017 & 0.008 & $4.40 \mathrm{E}-02$ & 36,603 & fat $\%$ \\
\hline rs11045172 & $12 / 20361488$ & $P D E 3 A$ & $\mathrm{~A} / \mathrm{C}$ & -0.015 & 0.007 & $1.83 \mathrm{E}-02$ & 123,259 & BMI \\
\hline rs11057405 & $12 / 121347850$ & CLIP1 & $\mathrm{A} / \mathrm{G}$ & 0.019 & 0.009 & $3.70 \mathrm{E}-02$ & 77,167 & WHR \\
\hline rs11057405 & $12 / 121347850$ & CLIPI & $\mathrm{A} / \mathrm{G}$ & -0.025 & 0.009 & $7.16 \mathrm{E}-03$ & 122,590 & BMI \\
\hline rs 2454722 & $12 / 121737171$ & GPR109A & $\mathrm{A} / \mathrm{G}$ & 0.018 & 0.006 & $4.00 \mathrm{E}-03$ & 77,167 & WHR \\
\hline rs601339 & $12 / 121740696$ & GPR109A & $\mathrm{A} / \mathrm{G}$ & 0.018 & 0.006 & $3.60 \mathrm{E}-03$ & 77,167 & WHR \\
\hline rs4759361 & $12 / 121744233$ & GPR109A & $\mathrm{A} / \mathrm{T}$ & -0.018 & 0.006 & $3.70 \mathrm{E}-03$ & 77,167 & WHR \\
\hline rs509548 & $12 / 121747808$ & GPR109A & $\mathrm{A} / \mathrm{T}$ & -0.018 & 0.006 & $3.70 \mathrm{E}-03$ & 77,167 & WHR \\
\hline rs6488898 & $12 / 122769785$ & ATP6V0A2 & $\mathrm{A} / \mathrm{G}$ & 0.023 & 0.010 & $1.72 \mathrm{E}-02$ & 119,083 & BMI \\
\hline rs11057354 & $12 / 122833591$ & DNAH10 & $\mathrm{A} / \mathrm{G}$ & 0.021 & 0.007 & $8.40 \mathrm{E}-03$ & 77,167 & WHR \\
\hline rs12317176 & $12 / 122970671$ & DNAH10 & $\mathrm{T} / \mathrm{C}$ & -0.015 & 0.005 & $2.24 \mathrm{E}-03$ & 123,864 & BMI \\
\hline rs12317176 & $12 / 122970671$ & DNAH10 & $\mathbf{T} / \mathbf{C}$ & 0.023 & 0.005 & $5.31 \mathrm{E}-06$ & 77,167 & WHR \\
\hline
\end{tabular}




\begin{tabular}{|c|c|c|c|c|c|c|c|c|}
\hline rs 12317176 & $12 / 122970671$ & DNAH10 & $\mathrm{T} / \mathrm{C}$ & -0.023 & 0.008 & $4.00 \mathrm{E}-03$ & 36,607 & fat $\%$ \\
\hline rs7301953 & $12 / 122971824$ & DNAH1O & $\mathrm{A} / \mathrm{G}$ & 0.014 & 0.005 & 7.03E-03 & 123,864 & BMI \\
\hline rs7301953 & $12 / 122971824$ & DNAH10 & $\mathrm{A} / \mathrm{G}$ & 0.026 & 0.008 & $1.00 \mathrm{E}-03$ & 36,593 & fat $\%$ \\
\hline rs 7301953 & $12 / 122971824$ & DNAH10 & $\mathbf{A} / \mathbf{G}$ & -0.022 & 0.005 & $1.50 \mathrm{E}-05$ & 77,167 & WHR \\
\hline rs10846579 & $12 / 122973356$ & DNAH10 & $\mathbf{T} / \mathbf{C}$ & -0.023 & 0.005 & $4.61 \mathrm{E}-06$ & 77,167 & WHR \\
\hline rs 10846579 & $12 / 122973356$ & DNAH10 & $\mathrm{T} / \mathrm{C}$ & 0.015 & 0.005 & $2.20 \mathrm{E}-03$ & 123,864 & BMI \\
\hline rs 10846579 & $12 / 122973356$ & DNAH10 & $\mathrm{T} / \mathrm{C}$ & 0.026 & 0.009 & $3.00 \mathrm{E}-03$ & 29,051 & fat $\%$ \\
\hline rs11057394 & $12 / 122973629$ & DNAH10 & $\mathbf{T} / \mathbf{C}$ & 0.023 & 0.005 & $3.60 \mathrm{E}-06$ & 77,167 & WHR \\
\hline rs11057394 & $12 / 122973629$ & DNAH10 & $\mathrm{T} / \mathrm{C}$ & -0.015 & 0.005 & $2.17 \mathrm{E}-03$ & 123,863 & BMI \\
\hline rs11057394 & $12 / 122973629$ & DNAH10 & $\mathrm{T} / \mathrm{C}$ & -0.024 & 0.008 & $3.00 \mathrm{E}-03$ & 36,607 & fat $\%$ \\
\hline rs12809125 & $12 / 122973944$ & DNAH10 & $\mathbf{A} / \mathbf{G}$ & 0.023 & 0.005 & $4.66 \mathrm{E}-06$ & 77,167 & WHR \\
\hline rs 12809125 & $12 / 122973944$ & DNAH10 & $\mathrm{A} / \mathrm{G}$ & -0.015 & 0.005 & $2.36 \mathrm{E}-03$ & 123,864 & BMI \\
\hline rs 12809125 & $12 / 122973944$ & DNAH10 & $\mathrm{A} / \mathrm{G}$ & -0.023 & 0.008 & $3.00 \mathrm{E}-03$ & 36,608 & fat $\%$ \\
\hline rs7133378 & $12 / 122975455$ & DNAH10 & $\mathrm{A} / \mathrm{G}$ & 0.016 & 0.005 & $1.70 \mathrm{E}-03$ & 123,863 & BMI \\
\hline rs7133378 & $12 / 122975455$ & DNAH10 & $\mathrm{A} / \mathrm{G}$ & 0.028 & 0.008 & $1.00 \mathrm{E}-03$ & 34,831 & fat $\%$ \\
\hline rs 7133378 & $12 / 122975455$ & $D N A H 10$ & $\mathbf{A} / \mathbf{G}$ & -0.022 & 0.005 & $2.38 \mathrm{E}-05$ & 77,167 & WHR \\
\hline rs9971695 & $12 / 122979444$ & DNAH10 & $\mathrm{A} / \mathrm{G}$ & 0.015 & 0.005 & $2.94 \mathrm{E}-03$ & 123,864 & BMI \\
\hline rs9971695 & $12 / 122979444$ & DNAH10 & $\mathrm{A} / \mathrm{G}$ & 0.023 & 0.008 & $3.00 \mathrm{E}-03$ & 36,608 & fat $\%$ \\
\hline rs9971695 & $12 / 122979444$ & DNAH10 & $\mathbf{A} / \mathbf{G}$ & -0.023 & 0.005 & $6.74 \mathrm{E}-06$ & 77,167 & WHR \\
\hline rs3802999 & $12 / 122980051$ & DNAH10 & $\mathbf{T} / \mathbf{C}$ & -0.023 & 0.005 & $5.87 \mathrm{E}-06$ & 77,167 & WHR \\
\hline rs3802999 & $12 / 122980051$ & DNAH10 & $\mathrm{T} / \mathrm{C}$ & 0.023 & 0.008 & $4.00 \mathrm{E}-03$ & 36,610 & fat $\%$ \\
\hline rs3802999 & $12 / 122980051$ & DNAHIO & $\mathrm{T} / \mathrm{C}$ & 0.015 & 0.005 & $2.95 \mathrm{E}-03$ & 123,864 & BMI \\
\hline rs4930721 & $12 / 122983842$ & DNAH10 & $\mathrm{T} / \mathrm{C}$ & 0.013 & 0.005 & $8.68 \mathrm{E}-03$ & 123,864 & BMI \\
\hline rs4930721 & $12 / 122983842$ & DNAH10 & $\mathrm{T} / \mathrm{C}$ & 0.025 & 0.008 & $2.00 \mathrm{E}-03$ & 36,600 & fat $\%$ \\
\hline rs4930721 & $12 / 122983842$ & DNAH10 & $\mathrm{T} / \mathrm{C}$ & -0.022 & 0.005 & $2.32 \mathrm{E}-05$ & 77,167 & WHR \\
\hline rs12298484 & $12 / 122984627$ & DNAH10 & $\mathrm{T} / \mathrm{C}$ & 0.023 & 0.008 & $4.00 \mathrm{E}-03$ & 36,597 & fat $\%$ \\
\hline rs 12298484 & $12 / 122984627$ & DNAH10 & $\mathrm{T} / \mathrm{C}$ & 0.015 & 0.005 & $2.16 \mathrm{E}-03$ & 123,789 & BMI \\
\hline rs12298484 & $12 / 122984627$ & DNAH10 & $\mathbf{T} / \mathbf{C}$ & -0.023 & 0.005 & $7.22 \mathrm{E}-06$ & 77,167 & WHR \\
\hline rs11057396 & $12 / 122985015$ & DNAH10 & $\mathrm{A} / \mathrm{C}$ & -0.014 & 0.007 & $3.95 \mathrm{E}-02$ & 67,240 & BMI \\
\hline rs11057397 & $12 / 122985681$ & DNAH10 & $\mathrm{T} / \mathrm{C}$ & 0.022 & 0.008 & $5.00 \mathrm{E}-03$ & 36,606 & fat $\%$ \\
\hline rs11057397 & $12 / 122985681$ & DNAH10 & $\mathbf{T} / \mathbf{C}$ & -0.022 & 0.005 & $9.34 \mathrm{E}-06$ & 77,167 & WHR \\
\hline rs11057397 & $12 / 122985681$ & DNAHIO & $\mathrm{T} / \mathrm{C}$ & 0.016 & 0.005 & $1.69 \mathrm{E}-03$ & 123,864 & BMI \\
\hline rs9863 & $12 / 122987406$ & CCDC92 & $\mathrm{T} / \mathrm{C}$ & -0.015 & 0.005 & $2.84 \mathrm{E}-03$ & 123,860 & BMI \\
\hline rs9863 & $12 / 122987406$ & $C C D C 92$ & $\mathrm{~T} / \mathrm{C}$ & -0.028 & 0.008 & $1.00 \mathrm{E}-03$ & 34,831 & fat $\%$ \\
\hline rs9863 & $12 / 122987406$ & CCDC92 & $\mathbf{T} / \mathbf{C}$ & 0.021 & 0.005 & $4.36 \mathrm{E}-05$ & 77,167 & WHR \\
\hline rs4930723 & $12 / 122989553$ & CCDC92 & $\mathrm{C} / \mathrm{G}$ & 0.022 & 0.008 & $5.00 \mathrm{E}-03$ & 36,613 & fat $\%$ \\
\hline rs4930723 & $12 / 122989553$ & CCDC92 & $\mathrm{C} / \mathrm{G}$ & 0.015 & 0.005 & $2.83 \mathrm{E}-03$ & 123,864 & BMI \\
\hline rs4930723 & $12 / 122989553$ & CCDC92 & $\mathbf{C} / \mathbf{G}$ & -0.022 & 0.005 & 8.89E-06 & 77,167 & WHR \\
\hline rs11057401 & $12 / 122993259$ & CCDC92 & $\mathrm{A} / \mathrm{T}$ & 0.024 & 0.008 & $3.00 \mathrm{E}-03$ & 36,528 & fat $\%$ \\
\hline rs11057401 & $12 / 122993259$ & $C C D C 92$ & $\mathrm{~A} / \mathrm{T}$ & 0.013 & 0.005 & $8.51 \mathrm{E}-03$ & 123,779 & BMI \\
\hline rs11057401 & $12 / 122993259$ & CCDC92 & $\mathbf{A} / \mathbf{T}$ & -0.021 & 0.005 & $3.77 \mathrm{E}-05$ & 77,167 & WHR \\
\hline rs4930726 & $12 / 122994284$ & CCDC92 & $\mathrm{T} / \mathrm{C}$ & -0.022 & 0.008 & $5.00 \mathrm{E}-03$ & 36,610 & fat $\%$ \\
\hline rs4930726 & $12 / 122994284$ & ССDC92 & $\mathrm{T} / \mathrm{C}$ & 0.022 & 0.005 & $1.16 \mathrm{E}-05$ & 77,167 & WHR \\
\hline rs4930726 & $12 / 122994284$ & CCDC92 & $\mathrm{T} / \mathrm{C}$ & -0.015 & 0.005 & $2.87 \mathrm{E}-03$ & 123,864 & BMI \\
\hline rs2178663 & $12 / 122999858$ & CCDC92 & $\mathrm{T} / \mathrm{C}$ & 0.013 & 0.005 & $8.68 \mathrm{E}-03$ & 123,864 & BMI \\
\hline rs2178663 & $12 / 122999858$ & CCDC92 & $\mathbf{T} / \mathbf{C}$ & -0.021 & 0.005 & 3.39E-05 & 77,167 & WHR \\
\hline rs 2178663 & $12 / 122999858$ & CCDC92 & $\mathrm{T} / \mathrm{C}$ & 0.024 & 0.008 & $2.00 \mathrm{E}-03$ & 36,601 & fat $\%$ \\
\hline rs 4405410 & $12 / 123001741$ & $C C D C 92$ & $\mathrm{~A} / \mathrm{T}$ & 0.022 & 0.008 & $6.00 \mathrm{E}-03$ & 36,610 & fat $\%$ \\
\hline rs 4405410 & $12 / 123001741$ & CCDC92 & $\mathrm{A} / \mathrm{T}$ & 0.015 & 0.005 & $3.00 \mathrm{E}-03$ & 123,864 & BMI \\
\hline rs4405410 & $12 / 123001741$ & CCDC 92 & $\mathbf{A} / \mathbf{T}$ & -0.022 & 0.005 & $1.23 \mathrm{E}-05$ & 77,167 & WHR \\
\hline rs7961449 & $12 / 123003168$ & CCDC 92 & $\mathbf{A} / \mathbf{T}$ & -0.022 & 0.005 & $1.23 \mathrm{E}-05$ & 77,167 & WHR \\
\hline rs7961449 & $12 / 123003168$ & CCDC92 & $\mathrm{A} / \mathrm{T}$ & 0.015 & 0.005 & $3.06 \mathrm{E}-03$ & 123,864 & BMI \\
\hline rs7961449 & $12 / 123003168$ & CCDC92 & $\mathrm{A} / \mathrm{T}$ & 0.022 & 0.008 & $6.00 \mathrm{E}-03$ & 36,610 & fat $\%$ \\
\hline
\end{tabular}




\begin{tabular}{|c|c|c|c|c|c|c|c|c|}
\hline rs3867146 & $12 / 123003958$ & $C C D C 92$ & $\mathrm{~A} / \mathrm{G}$ & 0.020 & 0.008 & $1.20 \mathrm{E}-02$ & 77,167 & WHR \\
\hline rs7132655 & $12 / 123004926$ & CCDC92 & $\mathrm{C} / \mathrm{G}$ & 0.015 & 0.005 & $3.41 \mathrm{E}-03$ & 123,864 & BMI \\
\hline rs7132655 & $12 / 123004926$ & $C C D C 92$ & $\mathrm{C} / \mathrm{G}$ & 0.021 & 0.008 & $7.00 \mathrm{E}-03$ & 36,608 & fat $\%$ \\
\hline rs7132655 & $12 / 123004926$ & CCDC92 & $\mathrm{C} / \mathrm{G}$ & -0.022 & 0.005 & $1.34 \mathrm{E}-05$ & 77,167 & WHR \\
\hline rs4765219 & $12 / 123006063$ & $C C D C 92$ & $\mathrm{~A} / \mathrm{C}$ & 0.015 & 0.005 & $3.04 \mathrm{E}-03$ & 123,849 & BMI \\
\hline rs4765219 & $12 / 123006063$ & CCDC92 & $\mathrm{A} / \mathrm{C}$ & 0.021 & 0.008 & 7.00E-03 & 36,606 & fat $\%$ \\
\hline rs4765219 & $12 / 123006063$ & CCDC 92 & $\mathbf{A} / \mathbf{C}$ & -0.022 & 0.005 & $1.53 E-05$ & 77,167 & WHR \\
\hline rs7958691 & $12 / 123006696$ & ССDC92 & $T / G$ & -0.022 & 0.005 & 1.40E-05 & 77,167 & WHR \\
\hline rs7958691 & $12 / 123006696$ & $C C D C 92$ & $\mathrm{~T} / \mathrm{G}$ & 0.025 & 0.009 & $3.00 \mathrm{E}-03$ & 29,051 & fat $\%$ \\
\hline rs7958691 & $12 / 123006696$ & CCDC92 & $\mathrm{T} / \mathrm{G}$ & 0.015 & 0.005 & $3.49 \mathrm{E}-03$ & 123,864 & BMI \\
\hline rs7305864 & $12 / 123007833$ & CCDC92 & $\mathbf{C} / \mathbf{G}$ & 0.022 & 0.005 & $1.61 \mathrm{E}-05$ & 77,167 & WHR \\
\hline rs7305864 & $12 / 123007833$ & CCDC92 & $\mathrm{C} / \mathrm{G}$ & -0.022 & 0.008 & $5.00 \mathrm{E}-03$ & 36,440 & fat $\%$ \\
\hline rs7305864 & $12 / 123007833$ & CCDC92 & $\mathrm{C} / \mathrm{G}$ & -0.014 & 0.005 & $3.88 \mathrm{E}-03$ & 123,680 & BMI \\
\hline rs6488913 & $12 / 123011522$ & CCDC92 & $\mathrm{C} / \mathrm{G}$ & -0.021 & 0.005 & 2.04E-05 & 77,167 & WHR \\
\hline rs6488913 & $12 / 123011522$ & CCDC92 & $\mathrm{C} / \mathrm{G}$ & 0.015 & 0.005 & $3.05 \mathrm{E}-03$ & 123,864 & BMI \\
\hline rs6488913 & $12 / 123011522$ & $C C D C 92$ & $\mathrm{C} / \mathrm{G}$ & 0.022 & 0.008 & $6.00 \mathrm{E}-03$ & 36,581 & fat $\%$ \\
\hline rs7312404 & $12 / 123012681$ & $C C D C 92$ & $\mathrm{~A} / \mathrm{G}$ & -0.025 & 0.011 & $1.90 \mathrm{E}-02$ & 18,010 & fat $\%$ \\
\hline rs7312404 & $12 / 123012681$ & CCDC92 & $\mathrm{A} / \mathrm{G}$ & -0.015 & 0.007 & $3.26 \mathrm{E}-02$ & 67,240 & BMI \\
\hline rs11831913 & $12 / 123012851$ & CCDC92 & $\mathrm{T} / \mathrm{C}$ & -0.013 & 0.005 & $1.01 \mathrm{E}-02$ & 123,864 & BMI \\
\hline rs11831913 & $12 / 123012851$ & CCDC92 & $\mathrm{T} / \mathrm{C}$ & -0.024 & 0.008 & $2.00 \mathrm{E}-03$ & 36,595 & fat $\%$ \\
\hline rs11831913 & $12 / 123012851$ & CCDC92 & $\mathbf{T} / \mathbf{C}$ & 0.021 & 0.005 & 5.38E-05 & 77,167 & WHR \\
\hline rs6488914 & $12 / 123013794$ & CCDC92 & $\mathrm{C} / \mathrm{G}$ & 0.024 & 0.008 & $2.00 \mathrm{E}-03$ & 36,602 & fat $\%$ \\
\hline rs6488914 & $12 / 123013794$ & $C C D C 92$ & $\mathrm{C} / \mathrm{G}$ & 0.013 & 0.005 & $9.36 \mathrm{E}-03$ & 123,841 & BMI \\
\hline rs6488914 & $12 / 123013794$ & ССDC92 & $\mathrm{C} / \mathrm{G}$ & -0.021 & 0.005 & $4.56 \mathrm{E}-05$ & 77,167 & WHR \\
\hline rs7973683 & $12 / 123015176$ & $C C D C 92$ & $\mathrm{~A} / \mathrm{C}$ & 0.015 & 0.005 & $3.06 \mathrm{E}-03$ & 123,831 & BMI \\
\hline rs7973683 & $12 / 123015176$ & CCDC92 & $\mathrm{A} / \mathrm{C}$ & 0.022 & 0.008 & $5.00 \mathrm{E}-03$ & 36,584 & fat $\%$ \\
\hline rs7973683 & $12 / 123015176$ & CCDC92 & $\mathbf{A} / \mathbf{C}$ & -0.022 & 0.005 & $1.98 E-05$ & 77,167 & WHR \\
\hline rs4765127 & $12 / 123026120$ & ZNF664 & $\mathrm{T} / \mathrm{G}$ & 0.023 & 0.008 & $4.00 \mathrm{E}-03$ & 36,504 & fat $\%$ \\
\hline rs4765127 & $12 / 123026120$ & ZNF664 & $\mathbf{T} / \mathbf{G}$ & -0.022 & 0.005 & $1.84 \mathrm{E}-05$ & 77,167 & WHR \\
\hline rs4765127 & $12 / 123026120$ & ZNF664 & $\mathrm{T} / \mathrm{G}$ & 0.016 & 0.005 & $1.70 \mathrm{E}-03$ & 123,737 & BMI \\
\hline rs12311114 & $12 / 123026656$ & ZNF664 & $\mathrm{A} / \mathrm{C}$ & 0.026 & 0.008 & $1.00 \mathrm{E}-03$ & 36,580 & fat $\%$ \\
\hline rs12311114 & $12 / 123026656$ & ZNF664 & $\mathrm{A} / \mathrm{C}$ & 0.014 & 0.005 & $4.70 \mathrm{E}-03$ & 123,847 & BMI \\
\hline rs12311114 & $12 / 123026656$ & ZNF664 & $\mathbf{A} / \mathbf{C}$ & -0.021 & 0.005 & 3.38E-05 & 77,167 & WHR \\
\hline rs4765528 & $12 / 123028207$ & ZNF664 & $\mathrm{A} / \mathrm{T}$ & 0.014 & 0.005 & $5.41 \mathrm{E}-03$ & 122,276 & BMI \\
\hline rs4765528 & $12 / 123028207$ & ZNF664 & $\mathrm{A} / \mathrm{T}$ & 0.026 & 0.008 & $2.00 \mathrm{E}-03$ & 34,681 & fat $\%$ \\
\hline rs4765528 & $12 / 123028207$ & ZNF664 & $\mathbf{A} / \mathbf{T}$ & -0.022 & 0.005 & 2.74E-05 & 77,167 & WHR \\
\hline rs11057408 & $12 / 123030789$ & ZNF664 & $\mathrm{T} / \mathrm{G}$ & 0.022 & 0.008 & $5.00 \mathrm{E}-03$ & 36,463 & fat $\%$ \\
\hline rs11057408 & $12 / 123030789$ & ZNF664 & $\mathbf{T} / \mathbf{G}$ & -0.021 & 0.005 & $2.40 \mathrm{E}-05$ & 77,167 & WHR \\
\hline rs11057408 & $12 / 123030789$ & ZNF664 & $\mathrm{T} / \mathrm{G}$ & 0.016 & 0.005 & $1.18 \mathrm{E}-03$ & 123,709 & BMI \\
\hline rs7978610 & $12 / 123034525$ & ZNF664 & $\mathrm{C} / \mathrm{G}$ & 0.016 & 0.005 & $1.06 \mathrm{E}-03$ & 123,835 & BMI \\
\hline rs7978610 & $12 / 123034525$ & ZNF664 & $\mathrm{C} / \mathrm{G}$ & 0.023 & 0.008 & $3.00 \mathrm{E}-03$ & 36,551 & fat $\%$ \\
\hline rs7978610 & $12 / 123034525$ & ZNF664 & $\mathbf{C} / \mathbf{G}$ & -0.022 & 0.005 & 1.04E-05 & 77,167 & WHR \\
\hline rs952632 & $12 / 123035691$ & ZNF664 & $\mathrm{A} / \mathrm{G}$ & -0.016 & 0.005 & $1.07 \mathrm{E}-03$ & 123,864 & BMI \\
\hline rs952632 & $12 / 123035691$ & ZNF664 & A/G & 0.022 & 0.005 & $1.40 \mathrm{E}-05$ & 77,167 & WHR \\
\hline rs952632 & $12 / 123035691$ & ZNF664 & $\mathrm{A} / \mathrm{G}$ & -0.024 & 0.008 & $2.00 \mathrm{E}-03$ & 36,613 & fat $\%$ \\
\hline rs11837287 & $12 / 123036012$ & ZNF664 & $\mathrm{T} / \mathrm{C}$ & 0.016 & 0.005 & $1.00 \mathrm{E}-03$ & 123,864 & BMI \\
\hline rs11837287 & $12 / 123036012$ & ZNF664 & $\mathbf{T} / \mathbf{C}$ & -0.022 & 0.005 & 1.29E-05 & 77,167 & WHR \\
\hline rs11837287 & $12 / 123036012$ & ZNF664 & $\mathrm{T} / \mathrm{C}$ & 0.023 & 0.008 & $3.00 \mathrm{E}-03$ & 36,619 & fat $\%$ \\
\hline rs7311969 & $12 / 123036286$ & ZNF664 & $\mathrm{T} / \mathrm{C}$ & -0.016 & 0.005 & $1.09 \mathrm{E}-03$ & 123,792 & BMI \\
\hline rs7311969 & $12 / 123036286$ & ZNF664 & $\mathbf{T} / \mathbf{C}$ & 0.023 & 0.005 & $4.51 \mathrm{E}-06$ & 77,167 & WHR \\
\hline rs7311969 & $12 / 123036286$ & ZNF664 & $\mathrm{T} / \mathrm{C}$ & -0.023 & 0.008 & $3.00 \mathrm{E}-03$ & 36,486 & fat $\%$ \\
\hline rs 7307277 & $12 / 123041109$ & ZNF664 & $\mathrm{A} / \mathrm{G}$ & -0.016 & 0.005 & $9.66 \mathrm{E}-04$ & 123,598 & BMI \\
\hline rs 7307277 & $12 / 123041109$ & ZNF664 & $\mathrm{A} / \mathrm{G}$ & -0.023 & 0.008 & $3.00 \mathrm{E}-03$ & 36,618 & fat $\%$ \\
\hline rs7307277 & 12/123041109 & ZNF664 & $\mathbf{A} / \mathbf{G}$ & 0.022 & 0.005 & $1.26 \mathrm{E}-05$ & 77,167 & WHR \\
\hline
\end{tabular}




\begin{tabular}{|c|c|c|c|c|c|c|c|c|}
\hline rs7311233 & $12 / 123041893$ & ZNF664 & $\mathrm{A} / \mathrm{G}$ & -0.026 & 0.008 & $1.00 \mathrm{E}-03$ & 36,599 & fat $\%$ \\
\hline rs7311233 & $12 / 123041893$ & ZNF664 & $\mathrm{A} / \mathrm{G}$ & -0.015 & 0.005 & $3.30 \mathrm{E}-03$ & 123,829 & BMI \\
\hline rs7311233 & $12 / 123041893$ & ZNF664 & $\mathbf{A} / \mathbf{G}$ & 0.021 & 0.005 & $3.48 \mathrm{E}-05$ & 77,167 & WHR \\
\hline rs 12809473 & $12 / 123042552$ & ZNF664 & $\mathrm{A} / \mathrm{T}$ & 0.017 & 0.005 & $9.44 \mathrm{E}-04$ & 123,864 & BMI \\
\hline rs12809473 & $12 / 123042552$ & ZNF664 & $\mathrm{A} / \mathrm{T}$ & 0.023 & 0.008 & $3.00 \mathrm{E}-03$ & 36,617 & fat $\%$ \\
\hline rs12809473 & $12 / 123042552$ & ZNF664 & $\mathbf{A} / \mathbf{T}$ & -0.022 & 0.005 & 1.52E-05 & 77,167 & WHR \\
\hline rs12827409 & $12 / 123042659$ & ZNF664 & $\mathrm{C} / \mathrm{G}$ & -0.023 & 0.008 & $3.00 \mathrm{E}-03$ & 36,618 & fat $\%$ \\
\hline rs12827409 & $12 / 123042659$ & ZNF664 & $\mathbf{C} / \mathbf{G}$ & 0.022 & 0.005 & $1.52 E-05$ & 77,167 & WHR \\
\hline rs12827409 & $12 / 123042659$ & ZNF664 & $\mathrm{C} / \mathrm{G}$ & -0.017 & 0.005 & $9.10 \mathrm{E}-04$ & 123,864 & BMI \\
\hline rs7135314 & $12 / 123044519$ & ZNF664 & $\mathrm{A} / \mathrm{T}$ & -0.023 & 0.008 & $3.00 \mathrm{E}-03$ & 36,618 & fat $\%$ \\
\hline rs7135314 & $12 / 123044519$ & ZNF664 & $\mathbf{A} / \mathbf{T}$ & 0.022 & 0.005 & $1.42 \mathrm{E}-05$ & 77,167 & WHR \\
\hline rs7135314 & $12 / 123044519$ & ZNF664 & $\mathrm{A} / \mathrm{T}$ & -0.016 & 0.005 & $9.61 \mathrm{E}-04$ & 123,864 & BMI \\
\hline rs4765148 & $12 / 123044590$ & ZNF664 & $\mathrm{T} / \mathrm{G}$ & 0.024 & 0.008 & $4.00 \mathrm{E}-03$ & 34,803 & fat $\%$ \\
\hline rs4765148 & $12 / 123044590$ & ZNF664 & $\mathrm{T} / \mathrm{G}$ & 0.015 & 0.005 & $4.09 \mathrm{E}-03$ & 123,835 & BMI \\
\hline rs4765148 & $12 / 123044590$ & ZNF664 & $\mathbf{T} / \mathbf{G}$ & -0.021 & 0.005 & $3.16 \mathrm{E}-05$ & 77,167 & WHR \\
\hline rs11057409 & $12 / 123045284$ & ZNF664 & $\mathrm{T} / \mathrm{C}$ & 0.016 & 0.005 & $1.17 \mathrm{E}-03$ & 123,784 & BMI \\
\hline rs11057409 & $12 / 123045284$ & ZNF664 & $\mathbf{T} / \mathbf{C}$ & -0.022 & 0.005 & $1.21 \mathrm{E}-05$ & 77,167 & WHR \\
\hline rs11057409 & $12 / 123045284$ & ZNF664 & $\mathrm{T} / \mathrm{C}$ & 0.024 & 0.008 & $3.00 \mathrm{E}-03$ & 36,583 & fat $\%$ \\
\hline rs7975482 & $12 / 123047643$ & ZNF664 & $\mathrm{A} / \mathrm{G}$ & -0.024 & 0.008 & $3.00 \mathrm{E}-03$ & 36,585 & fat $\%$ \\
\hline rs7975482 & $12 / 123047643$ & ZNF664 & $\mathbf{A} / \mathbf{G}$ & 0.022 & 0.005 & $1.38 \mathrm{E}-05$ & 77,167 & WHR \\
\hline rs7975482 & $12 / 123047643$ & ZNF664 & $\mathrm{A} / \mathrm{G}$ & -0.017 & 0.005 & $9.91 \mathrm{E}-04$ & 123,837 & BMI \\
\hline rs2130382 & $12 / 123050596$ & ZNF664 & $\mathbf{C} / \mathbf{G}$ & 0.022 & 0.005 & 2.92E-05 & 77,167 & WHR \\
\hline rs 2130382 & $12 / 123050596$ & ZNF664 & $\mathrm{C} / \mathrm{G}$ & -0.015 & 0.005 & $3.56 \mathrm{E}-03$ & 123,860 & BMI \\
\hline rs2130382 & $12 / 123050596$ & ZNF664 & $\mathrm{C} / \mathrm{G}$ & -0.025 & 0.008 & $2.00 \mathrm{E}-03$ & 36,622 & fat $\%$ \\
\hline rs12310367 & $12 / 123052631$ & ZNF664 & $\mathbf{A} / \mathbf{G}$ & 0.022 & 0.005 & 1.72E-05 & 77,167 & WHR \\
\hline rs 12310367 & $12 / 123052631$ & ZNF664 & $\mathrm{A} / \mathrm{G}$ & -0.017 & 0.005 & $8.35 \mathrm{E}-04$ & 123,859 & BMI \\
\hline rs 12310367 & $12 / 123052631$ & ZNF664 & $\mathrm{A} / \mathrm{G}$ & -0.025 & 0.008 & $2.00 \mathrm{E}-03$ & 36,612 & fat $\%$ \\
\hline rs11057412 & $12 / 123055095$ & ZNF664 & $\mathbf{C} / \mathbf{G}$ & -0.022 & 0.005 & $1.71 \mathrm{E}-05$ & 77,167 & WHR \\
\hline rs11057412 & $12 / 123055095$ & ZNF664 & $\mathrm{C} / \mathrm{G}$ & 0.017 & 0.005 & 7.97E-04 & 123,859 & BMI \\
\hline rs11057412 & $12 / 123055095$ & ZNF664 & $\mathrm{C} / \mathrm{G}$ & 0.030 & 0.009 & $1.00 \mathrm{E}-03$ & 29,056 & fat $\%$ \\
\hline rs1187415 & $12 / 123057482$ & ZNF664 & $\mathrm{C} / \mathrm{G}$ & -0.023 & 0.008 & $4.00 \mathrm{E}-03$ & 36,551 & fat $\%$ \\
\hline rs1187415 & $12 / 123057482$ & ZNF664 & $\mathbf{C} / \mathbf{G}$ & 0.023 & 0.005 & 8.41E-06 & 77,167 & WHR \\
\hline rs1187415 & $12 / 123057482$ & ZNF664 & $\mathrm{C} / \mathrm{G}$ & -0.017 & 0.005 & 7.99E-04 & 123,849 & BMI \\
\hline rs12303671 & $12 / 123058563$ & ZNF664 & $\mathrm{T} / \mathrm{G}$ & -0.024 & 0.008 & $3.00 \mathrm{E}-03$ & 36,615 & fat $\%$ \\
\hline rs12303671 & $12 / 123058563$ & ZNF664 & $\mathrm{T} / \mathrm{G}$ & -0.017 & 0.005 & $8.55 \mathrm{E}-04$ & 123,859 & BMI \\
\hline rs12303671 & $12 / 123058563$ & ZNF664 & $\mathbf{T} / \mathbf{G}$ & 0.022 & 0.005 & $1.92 \mathrm{E}-05$ & 77,167 & WHR \\
\hline rs7307053 & $12 / 123060493$ & ZNF664 & $\mathbf{T} / \mathbf{C}$ & -0.021 & 0.005 & 4.58E-05 & 77,167 & WHR \\
\hline rs7307053 & $12 / 123060493$ & ZNF664 & $\mathrm{T} / \mathrm{C}$ & 0.026 & 0.008 & $2.00 \mathrm{E}-03$ & 36,617 & fat $\%$ \\
\hline rs7307053 & $12 / 123060493$ & ZNF664 & $\mathrm{T} / \mathrm{C}$ & 0.015 & 0.005 & $3.56 \mathrm{E}-03$ & 123,851 & BMI \\
\hline rs 12824567 & $12 / 123061156$ & ZNF664 & $\mathrm{C} / \mathrm{G}$ & 0.015 & 0.005 & $3.40 \mathrm{E}-03$ & 123,857 & BMI \\
\hline rs12824567 & $12 / 123061156$ & ZNF664 & $\mathbf{C} / \mathbf{G}$ & -0.021 & 0.005 & 3.93E-05 & 77,167 & WHR \\
\hline rs 12824567 & $12 / 123061156$ & ZNF664 & $\mathrm{C} / \mathrm{G}$ & 0.026 & 0.008 & $2.00 \mathrm{E}-03$ & 36,623 & fat $\%$ \\
\hline rs863750 & $12 / 123071397$ & ZNF664 & $\mathbf{T} / \mathbf{C}$ & 0.020 & 0.005 & 7.67E-05 & 77,167 & WHR \\
\hline rs10773049 & $12 / 123072584$ & ZNF664 & $\mathbf{T} / \mathbf{C}$ & 0.018 & 0.005 & 4.50E-04 & 77,167 & WHR \\
\hline rs 825453 & $12 / 123074711$ & ZNF664 & $\mathrm{A} / \mathrm{T}$ & -0.017 & 0.005 & $6.80 \mathrm{E}-04$ & 77,167 & WHR \\
\hline rs11057418 & $12 / 123074929$ & ZNF664 & $\mathrm{C} / \mathrm{G}$ & 0.020 & 0.006 & $1.10 \mathrm{E}-03$ & 77,167 & WHR \\
\hline rs11057418 & $12 / 123074929$ & ZNF664 & $\mathrm{C} / \mathrm{G}$ & -0.017 & 0.006 & $7.50 \mathrm{E}-03$ & 120,031 & BMI \\
\hline rs11057418 & $12 / 123074929$ & ZNF664 & $\mathrm{C} / \mathrm{G}$ & -0.031 & 0.010 & $2.00 \mathrm{E}-03$ & 35,427 & fat $\%$ \\
\hline rs2927328 & $16 / 80067223$ & $C M I P$ & $\mathrm{~T} / \mathrm{C}$ & -0.018 & 0.008 & $2.50 \mathrm{E}-02$ & 34,832 & fat $\%$ \\
\hline rs 1471379 & $16 / 80068200$ & CMIP & $\mathrm{T} / \mathrm{C}$ & -0.017 & 0.008 & $2.10 \mathrm{E}-02$ & 36,550 & fat $\%$ \\
\hline rs1966957 & $16 / 80068580$ & $C M I P$ & $\mathrm{C} / \mathrm{G}$ & 0.018 & 0.008 & $1.90 \mathrm{E}-02$ & 36,560 & fat $\%$ \\
\hline rs 2927327 & $16 / 80069035$ & CMIP & $\mathrm{A} / \mathrm{T}$ & 0.017 & 0.008 & $2.00 \mathrm{E}-02$ & 36,563 & fat $\%$ \\
\hline rs2966079 & $16 / 80069307$ & $C M I P$ & $\mathrm{~T} / \mathrm{C}$ & 0.015 & 0.007 & $4.20 \mathrm{E}-02$ & 36,578 & fat $\%$ \\
\hline rs1471152 & $16 / 80070137$ & CMIP & $\mathrm{T} / \mathrm{G}$ & -0.016 & 0.007 & $3.10 \mathrm{E}-02$ & 36,561 & fat $\%$ \\
\hline
\end{tabular}




\begin{tabular}{|c|c|c|c|c|c|c|c|c|}
\hline rs2927323 & $16 / 80070449$ & CMIP & $\mathrm{A} / \mathrm{G}$ & 0.021 & 0.008 & $9.00 \mathrm{E}-03$ & 36,603 & fat $\%$ \\
\hline rs2927323 & $16 / 80070449$ & CMIP & $\mathrm{A} / \mathrm{G}$ & -0.011 & 0.005 & $3.50 \mathrm{E}-02$ & 77,167 & WHR \\
\hline rs2927322 & $16 / 80072006$ & CMIP & $\mathrm{A} / \mathrm{G}$ & 0.012 & 0.005 & $2.70 \mathrm{E}-02$ & 77,167 & WHR \\
\hline rs2927322 & $16 / 80072006$ & CMIP & $\mathrm{A} / \mathrm{G}$ & -0.018 & 0.009 & $3.90 \mathrm{E}-02$ & 34,831 & fat $\%$ \\
\hline rs2966085 & $16 / 80074135$ & $C M I P$ & $\mathrm{~A} / \mathrm{G}$ & -0.018 & 0.008 & $1.90 \mathrm{E}-02$ & 36,463 & fat $\%$ \\
\hline rs2317241 & $16 / 80077267$ & CMIP & $\mathrm{A} / \mathrm{G}$ & -0.022 & 0.008 & $1.00 \mathrm{E}-02$ & 34,831 & fat $\%$ \\
\hline rs12443634 & $16 / 80081775$ & CMIP & $\mathrm{A} / \mathrm{C}$ & 0.013 & 0.005 & $2.00 \mathrm{E}-02$ & 77,167 & WHR \\
\hline rs12443634 & $16 / 80081775$ & CMIP & $\mathrm{A} / \mathrm{C}$ & -0.020 & 0.009 & $2.40 \mathrm{E}-02$ & 34,831 & fat $\%$ \\
\hline rs2925979 & 16/80092291 & CMIP & $\mathrm{T} / \mathrm{C}$ & 0.013 & 0.005 & $1.50 \mathrm{E}-02$ & 77,167 & WHR \\
\hline rs2925979 & $16 / 80092291$ & CMIP & $\mathrm{T} / \mathrm{C}$ & -0.018 & 0.009 & $3.00 \mathrm{E}-02$ & 34,832 & fat $\%$ \\
\hline rs2966093 & $16 / 80096121$ & CMIP & $\mathrm{A} / \mathrm{G}$ & -0.011 & 0.005 & $3.10 \mathrm{E}-02$ & 77,167 & WHR \\
\hline rs2966093 & $16 / 80096121$ & CMIP & $\mathrm{A} / \mathrm{G}$ & 0.017 & 0.008 & $3.00 \mathrm{E}-02$ & 36,620 & fat $\%$ \\
\hline rs2966094 & $16 / 80096138$ & $C M I P$ & $\mathrm{~A} / \mathrm{C}$ & 0.012 & 0.005 & $2.40 \mathrm{E}-02$ & 77,167 & WHR \\
\hline rs2966094 & $16 / 80096138$ & CMIP & $\mathrm{A} / \mathrm{C}$ & -0.018 & 0.008 & $2.90 \mathrm{E}-02$ & 36,562 & fat $\%$ \\
\hline rs2966095 & $16 / 80106959$ & CMIP & $\mathrm{A} / \mathrm{G}$ & -0.019 & 0.008 & $2.20 \mathrm{E}-02$ & 36,543 & fat $\%$ \\
\hline rs889140 & $19 / 38580840$ & $P E P D$ & $\mathrm{~A} / \mathrm{G}$ & 0.013 & 0.005 & $7.98 \mathrm{E}-03$ & 123,845 & BMI \\
\hline rs889139 & $19 / 38581209$ & $P E P D$ & $\mathrm{~A} / \mathrm{G}$ & -0.014 & 0.005 & $6.03 \mathrm{E}-03$ & 123,846 & BMI \\
\hline rs731839 & $19 / 38590905$ & PEPD & $\mathrm{A} / \mathrm{G}$ & 0.014 & 0.005 & $5.20 \mathrm{E}-03$ & 123,854 & BMI \\
\hline rs731839 & $19 / 38590905$ & PEPD & $\mathrm{A} / \mathrm{G}$ & 0.018 & 0.008 & $2.70 \mathrm{E}-02$ & 34,832 & fat $\%$ \\
\hline rs4805885 & $19 / 38597963$ & PEPD & $\mathrm{T} / \mathrm{C}$ & -0.016 & 0.005 & $1.65 \mathrm{E}-03$ & 123,859 & BMI \\
\hline rs8182584 & $19 / 38601550$ & $P E P D$ & $\mathrm{~T} / \mathrm{G}$ & -0.016 & 0.005 & $1.39 \mathrm{E}-03$ & 123,858 & BMI \\
\hline
\end{tabular}

The beta expressed in inverse normally transformed BMI units (i.e. interpretable as SD or $\mathrm{Z}$-score), shows the change in BMI per additional effect allele.

* Results that are statistically significant, accounting for the number of independent SNPs, are highlighted in bold.

EA: Effect Allele

NEA: Non-Effect Allele

EA-Freq: Frequency of Effect Allele 


\section{Table S7 \\ D) Association results of nominally significant SNPs with lipid traits in the GLGC Consortium.}

\begin{tabular}{|c|c|c|c|c|c|c|c|c|}
\hline SNP & Chr/position & Closest Gene & EA/NEA & Beta & SE & $\mathbf{P}$ & $\mathbf{N}$ & associated traits \\
\hline rs13081028 & $3 / 52530356$ & $S T A B 1$ & $\mathbf{A} / \mathbf{G}$ & 0.018 & 0.005 & 3.53E-04 & 99,900 & HDL-C \\
\hline rs13081028 & $3 / 52530356$ & STABI & $\mathrm{A} / \mathrm{G}$ & -0.015 & 0.005 & $1.00 \mathrm{E}-03$ & 96,598 & TG \\
\hline rs9853056 & $3 / 52530997$ & $S T A B 1$ & $\mathrm{~T} / \mathrm{C}$ & -0.018 & 0.005 & 3.08E-04 & 99,900 & HDL-C \\
\hline rs9853056 & $3 / 52530997$ & STABI & $\mathrm{T} / \mathrm{C}$ & 0.015 & 0.005 & $9.35 \mathrm{E}-04$ & 96,598 & TG \\
\hline rs4282054 & $3 / 52541105$ & NT5DC2 & $\mathrm{T} / \mathrm{C}$ & -0.018 & 0.005 & 3.38E-04 & 99,900 & HDL-C \\
\hline rs4282054 & $3 / 52541105$ & $N T 5 D C 2$ & $\mathrm{~T} / \mathrm{C}$ & 0.015 & 0.005 & $8.75 \mathrm{E}-04$ & 96,598 & TG \\
\hline rs2590838 & $3 / 52597126$ & PBRMI & $\mathrm{A} / \mathrm{G}$ & 0.011 & 0.005 & $2.54 \mathrm{E}-02$ & 99,900 & HDL-C \\
\hline rs2590838 & $3 / 52597126$ & PBRMI & $\mathrm{A} / \mathrm{G}$ & -0.011 & 0.005 & $2.20 \mathrm{E}-02$ & 96,598 & TG \\
\hline rs9879090 & $3 / 52623305$ & PBRMI & $\mathrm{T} / \mathrm{C}$ & 0.012 & 0.005 & $2.96 \mathrm{E}-02$ & 92,418 & TG \\
\hline rs13083798 & $3 / 52624788$ & PBRMI & $\mathrm{A} / \mathrm{G}$ & -0.010 & 0.005 & 2.82E-02 & 99,877 & HDL-C \\
\hline rs13083798 & $3 / 52624788$ & PBRMI & $\mathrm{A} / \mathrm{G}$ & 0.011 & 0.005 & $2.30 \mathrm{E}-02$ & 96,575 & TG \\
\hline rs1108842 & $3 / 52695120$ & $G N L 3$ & $\mathrm{~A} / \mathrm{C}$ & -0.012 & 0.005 & $8.00 \mathrm{E}-03$ & 99,900 & HDL-C \\
\hline rs1108842 & $3 / 52695120$ & GNL3 & $\mathrm{A} / \mathrm{C}$ & 0.013 & 0.005 & $6.95 \mathrm{E}-03$ & 96,598 & TG \\
\hline rs4481150 & $3 / 52812833$ & ITIH3 & $\mathrm{T} / \mathrm{C}$ & -0.009 & 0.005 & $4.50 \mathrm{E}-02$ & 99,900 & HDL-C \\
\hline rs7636643 & $3 / 151532470$ & TSC22D2 & $\mathrm{A} / \mathrm{T}$ & 0.021 & 0.009 & $7.54 \mathrm{E}-03$ & 99,883 & HDL-C \\
\hline rs7636643 & $3 / 151532470$ & $T S C 22 D 2$ & $\mathrm{~A} / \mathrm{T}$ & -0.025 & 0.008 & $3.61 \mathrm{E}-03$ & 96,582 & TG \\
\hline rs16862607 & $3 / 151533988$ & $T S C 22 D 2$ & $\mathrm{~A} / \mathrm{G}$ & 0.021 & 0.009 & $8.71 \mathrm{E}-03$ & 99,900 & HDL-C \\
\hline rs16862607 & $3 / 151533988$ & $T S C 22 D 2$ & $\mathrm{~A} / \mathrm{G}$ & -0.024 & 0.008 & $5.56 \mathrm{E}-03$ & 96,598 & TG \\
\hline rs 16862610 & $3 / 151535497$ & TSC22D2 & $\mathrm{T} / \mathrm{C}$ & 0.020 & 0.009 & $1.32 \mathrm{E}-02$ & 99,879 & HDL-C \\
\hline rs 16862610 & $3 / 151535497$ & $T S C 22 D 2$ & $\mathrm{~T} / \mathrm{C}$ & -0.024 & 0.008 & $3.73 \mathrm{E}-03$ & 96,577 & TG \\
\hline rs2060662 & $3 / 151536642$ & TSC22D2 & $\mathrm{C} / \mathrm{G}$ & 0.020 & 0.009 & $1.20 \mathrm{E}-02$ & 99,900 & HDL-C \\
\hline rs2060662 & $3 / 151536642$ & $T S C 22 D 2$ & $\mathrm{C} / \mathrm{G}$ & -0.024 & 0.008 & $4.51 \mathrm{E}-03$ & 96,598 & TG \\
\hline rs1597466 & $3 / 151538251$ & $T S C 22 D 2$ & $\mathrm{~T} / \mathrm{G}$ & -0.020 & 0.009 & $1.20 \mathrm{E}-02$ & 99,900 & HDL-C \\
\hline rs1597466 & $3 / 151538251$ & $T S C 22 D 2$ & $\mathrm{~T} / \mathrm{G}$ & 0.024 & 0.008 & $3.74 \mathrm{E}-03$ & 96,598 & TG \\
\hline rs7616723 & $3 / 151538334$ & TSC22D2 & $\mathrm{A} / \mathrm{G}$ & -0.021 & 0.009 & $9.78 \mathrm{E}-03$ & 99,900 & HDL-C \\
\hline rs7616723 & $3 / 151538334$ & TSC22D2 & $\mathrm{A} / \mathrm{G}$ & 0.023 & 0.008 & $5.66 \mathrm{E}-03$ & 96,598 & TG \\
\hline rs7617025 & $3 / 151538648$ & TSC22D2 & $\mathrm{A} / \mathrm{G}$ & -0.020 & 0.009 & $1.01 \mathrm{E}-02$ & 99,900 & HDL-C \\
\hline rs7617025 & $3 / 151538648$ & TSC22D2 & $\mathrm{A} / \mathrm{G}$ & 0.024 & 0.008 & $4.35 \mathrm{E}-03$ & 96,598 & TG \\
\hline rs11924390 & $3 / 187915793$ & $K N G 1$ & $\mathrm{~T} / \mathrm{C}$ & 0.010 & 0.005 & $3.91 \mathrm{E}-02$ & 99,900 & HDL-C \\
\hline rs2062632 & $3 / 187943875$ & $K N G 1$ & $\mathrm{~T} / \mathrm{C}$ & 0.012 & 0.006 & $4.39 \mathrm{E}-02$ & 99,900 & HDL-C \\
\hline rs 266760 & $3 / 187943910$ & $K N G 1$ & $\mathrm{~A} / \mathrm{G}$ & -0.024 & 0.011 & $2.68 \mathrm{E}-02$ & 36,612 & HDL-C \\
\hline rs 266760 & $3 / 187943910$ & $K N G 1$ & $\mathrm{~A} / \mathrm{G}$ & 0.017 & 0.009 & $1.71 \mathrm{E}-02$ & 33,198 & TG \\
\hline rs1354091 & $3 / 187988594$ & EIF $4 A 2$ & $\mathrm{~T} / \mathrm{G}$ & 0.007 & 0.006 & 4.47E-02 & 99,888 & HDL-C \\
\hline rs2066500 & $3 / 187990616$ & $R F C 4$ & $\mathrm{~T} / \mathrm{C}$ & -0.008 & 0.006 & $4.09 \mathrm{E}-02$ & 99,891 & HDL-C \\
\hline rs3917110 & $3 / 188001346$ & $R F C 4$ & $\mathrm{C} / \mathrm{G}$ & 0.008 & 0.006 & $3.42 \mathrm{E}-02$ & 99,900 & HDL-C \\
\hline rs16861184 & $3 / 188003171$ & $R F C 4$ & $\mathrm{~T} / \mathrm{C}$ & -0.008 & 0.006 & $4.10 \mathrm{E}-02$ & 99,900 & HDL-C \\
\hline rs2293243 & $3 / 188005431$ & $R F C 4$ & $\mathrm{~A} / \mathrm{T}$ & 0.009 & 0.006 & $3.06 \mathrm{E}-02$ & 99,900 & HDL-C \\
\hline rs2954018 & $8 / 126546335$ & TRIB1 & $\mathrm{T} / \mathrm{C}$ & 0.040 & 0.005 & $1.93 E-13$ & 99,864 & HDL-C \\
\hline rs2954018 & $8 / 126546335$ & TRIBI & $\mathrm{T} / \mathrm{C}$ & -0.038 & 0.006 & 2.05E-11 & 95,418 & LDL-C \\
\hline rs2954018 & $8 / 126546335$ & TRIBI & $\mathrm{T} / \mathrm{C}$ & -0.042 & 0.006 & $3.60 \mathrm{E}-14$ & 100,147 & TC \\
\hline rs2954018 & $8 / 126546335$ & TRIB1 & $\mathrm{T} / \mathrm{C}$ & -0.061 & 0.005 & 2.35E-31 & 96,561 & TG \\
\hline rs2980880 & $8 / 126550154$ & TRIB1 & $\mathbf{A} / \mathbf{G}$ & 0.038 & 0.005 & $7.92 \mathrm{E}-13$ & 99,900 & HDL-C \\
\hline rs2980880 & $8 / 126550154$ & TRIB1 & A/G & -0.037 & 0.005 & 8.50E-12 & 95,454 & LDL-C \\
\hline rs2980880 & $8 / 126550154$ & TRIB1 & $\mathbf{A} / \mathbf{G}$ & -0.041 & 0.005 & $1.08 E-14$ & 100,184 & TC \\
\hline rs2980880 & $8 / 126550154$ & TRIB1 & A/G & -0.058 & 0.005 & 3.01E-30 & 96,598 & TG \\
\hline rs2980879 & $8 / 126550657$ & TRIBI & $\mathbf{A} / \mathbf{T}$ & -0.039 & 0.005 & 2.93E-13 & 99,900 & HDL-C \\
\hline rs2980879 & $8 / 126550657$ & TRIB1 & $\mathbf{A} / \mathbf{T}$ & 0.038 & 0.005 & $3.12 \mathrm{E}-12$ & 95,454 & LDL-C \\
\hline
\end{tabular}




\begin{tabular}{|c|c|c|c|c|c|c|c|c|}
\hline rs2980879 & $8 / 126550657$ & TRIBI & $\mathbf{A} / \mathbf{T}$ & 0.041 & 0.005 & $9.96 \mathrm{E}-15$ & 100,184 & $\mathbf{T C}$ \\
\hline rs2980879 & $8 / 126550657$ & $T R I B 1$ & $\mathbf{A} / \mathbf{T}$ & 0.058 & 0.005 & $1.58 \mathrm{E}-29$ & 96,598 & TG \\
\hline rs2980878 & $8 / 126550709$ & TRIB1 & $\mathrm{C} / \mathrm{G}$ & 0.043 & 0.005 & $2.22 \mathrm{E}-15$ & 96,908 & HDL-C \\
\hline rs2980878 & $8 / 126550709$ & $T R I B 1$ & $\mathrm{C} / \mathrm{G}$ & -0.036 & 0.006 & 8.88E-11 & 92,503 & LDL-C \\
\hline rs2980878 & $8 / 126550709$ & $T R I B 1$ & $\mathrm{C} / \mathrm{G}$ & -0.039 & 0.006 & $2.93 \mathrm{E}-12$ & 97,148 & TC \\
\hline rs2980878 & $8 / 126550709$ & $T R I B 1$ & $\mathrm{C} / \mathrm{G}$ & -0.058 & 0.005 & $1.16 \mathrm{E}-28$ & 93,562 & TG \\
\hline rs2980876 & $8 / 126550876$ & $T R I B 1$ & $\mathbf{T} / \mathbf{C}$ & 0.038 & 0.005 & $1.24 \mathrm{E}-12$ & 99,900 & HDL-C \\
\hline rs2980876 & $8 / 126550876$ & $T R I B 1$ & $\mathbf{T} / \mathbf{C}$ & -0.037 & 0.005 & 8.75E-12 & 95,454 & LDL-C \\
\hline rs2980876 & $8 / 126550876$ & $T R I B 1$ & $\mathbf{T} / \mathbf{C}$ & -0.041 & 0.005 & $1.25 \mathrm{E}-14$ & 100,184 & TC \\
\hline rs2980876 & $8 / 126550876$ & $T R I B 1$ & $\mathbf{T} / \mathbf{C}$ & -0.057 & 0.005 & $1.19 E-29$ & 96,598 & TG \\
\hline rs2954026 & $8 / 126553708$ & $T R I B 1$ & $\mathbf{T} / \mathbf{G}$ & -0.041 & 0.005 & $2.20 \mathrm{E}-14$ & 99,900 & HDL-C \\
\hline rs2954026 & $8 / 126553708$ & $T R I B 1$ & $\mathbf{T} / \mathbf{G}$ & 0.037 & 0.006 & $2.26 \mathrm{E}-11$ & 95,454 & LDL-C \\
\hline rs2954026 & $8 / 126553708$ & $T R I B 1$ & $\mathbf{T} / \mathbf{G}$ & 0.041 & 0.006 & 4.37E-14 & 100,184 & TC \\
\hline rs2954026 & $8 / 126553708$ & $T R I B 1$ & $\mathbf{T} / \mathbf{G}$ & 0.060 & 0.005 & $1.48 \mathrm{E}-31$ & 96,598 & TG \\
\hline rs7846466 & $8 / 126554713$ & $T R I B 1$ & $\mathbf{T} / \mathbf{C}$ & -0.042 & 0.005 & $6.96 \mathrm{E}-15$ & 96,908 & HDL-C \\
\hline rs7846466 & $8 / 126554713$ & $T R I B 1$ & $\mathbf{T} / \mathbf{C}$ & 0.036 & 0.006 & $1.66 \mathrm{E}-10$ & 92,503 & LDL-C \\
\hline rs7846466 & $8 / 126554713$ & $T R I B 1$ & $\mathbf{T} / \mathbf{C}$ & 0.039 & 0.006 & $1.53 E-12$ & 97,148 & TC \\
\hline rs7846466 & $8 / 126554713$ & $T R I B 1$ & $\mathbf{T} / \mathbf{C}$ & 0.060 & 0.005 & $4.90 \mathrm{E}-30$ & 93,562 & TG \\
\hline rs2954032 & $8 / 126562574$ & $T R I B 1$ & $\mathbf{A} / \mathbf{G}$ & -0.041 & 0.005 & 3.93E-14 & $\mathbf{9 9 , 8 7 7}$ & HDL-C \\
\hline rs2954032 & $8 / 126562574$ & $T R I B 1$ & $\mathrm{~A} / \mathrm{G}$ & 0.038 & 0.006 & $9.99 E-12$ & 95,433 & LDL-C \\
\hline rs2954032 & $8 / 126562574$ & $T R I B 1$ & $\mathbf{A} / \mathbf{G}$ & 0.041 & 0.006 & $4.70 \mathrm{E}-14$ & 100,161 & TC \\
\hline rs2954032 & $8 / 126562574$ & $T R I B 1$ & $\mathbf{A} / \mathbf{G}$ & 0.061 & 0.005 & $6.18 E-32$ & 96,575 & TG \\
\hline rs2954033 & $8 / 126562928$ & $T R I B 1$ & $\mathbf{A} / \mathbf{G}$ & -0.041 & 0.005 & $4.95 \mathrm{E}-14$ & 99,900 & HDL-C \\
\hline rs2954033 & $8 / 126562928$ & $T R I B 1$ & $\mathbf{A} / \mathbf{G}$ & 0.037 & 0.006 & $3.34 \mathrm{E}-11$ & 95,454 & LDL-C \\
\hline rs2954033 & $8 / 126562928$ & $T R I B 1$ & $\mathbf{A} / \mathbf{G}$ & 0.042 & 0.006 & $4.47 E-14$ & 100,184 & TC \\
\hline rs2954033 & $8 / 126562928$ & $T R I B 1$ & $\mathrm{~A} / \mathrm{G}$ & 0.062 & 0.005 & $3.25 \mathrm{E}-32$ & 96,598 & TG \\
\hline rs2980859 & $8 / 126568611$ & TRIB1 & $\mathbf{C} / \mathbf{G}$ & -0.042 & 0.005 & 2.33E-14 & $\mathbf{9 9 , 9 0 0}$ & HDL-C \\
\hline rs2980859 & $8 / 126568611$ & $T R I B 1$ & $\mathrm{C} / \mathrm{G}$ & 0.038 & 0.006 & $3.87 \mathrm{E}-11$ & 95,454 & LDL-C \\
\hline rs2980859 & $8 / 126568611$ & $T R I B 1$ & $\mathrm{C} / \mathrm{G}$ & 0.040 & 0.006 & $6.34 \mathrm{E}-13$ & 100,184 & TC \\
\hline rs2980859 & $8 / 126568611$ & $T R I B 1$ & $\mathrm{C} / \mathrm{G}$ & 0.060 & 0.005 & 5.98E-29 & 96,598 & TG \\
\hline rs2980888 & $8 / 126576490$ & $T R I B 1$ & $\mathbf{T} / \mathbf{C}$ & -0.041 & 0.006 & $7.83 \mathrm{E}-12$ & 99,900 & HDL-C \\
\hline rs2980888 & $8 / 126576490$ & TRIB1 & $\mathbf{T} / \mathbf{C}$ & 0.042 & 0.006 & $6.97 E-12$ & 95,454 & LDL-C \\
\hline rs2980888 & $8 / 126576490$ & $T R I B 1$ & $\mathbf{T} / \mathbf{C}$ & 0.048 & 0.006 & $1.56 \mathrm{E}-15$ & 100,184 & TC \\
\hline rs2980888 & $8 / 126576490$ & $T R I B 1$ & $\mathbf{T} / \mathbf{C}$ & 0.067 & 0.006 & $1.61 \mathrm{E}-31$ & 96,598 & TG \\
\hline rs2954038 & $8 / 126576571$ & $T R I B 1$ & $\mathbf{A} / \mathbf{C}$ & 0.041 & 0.006 & $7.94 \mathrm{E}-12$ & 99,900 & HDL-C \\
\hline rs2954038 & $8 / 126576571$ & $T R I B 1$ & $\mathrm{~A} / \mathrm{C}$ & -0.043 & 0.006 & $3.44 \mathrm{E}-12$ & 95,454 & LDL-C \\
\hline rs2954038 & $8 / 126576571$ & $T R I B 1$ & $\mathbf{A} / \mathbf{C}$ & -0.049 & 0.006 & $5.76 \mathrm{E}-16$ & 100,184 & TC \\
\hline rs2954038 & $8 / 126576571$ & $T R I B 1$ & $\mathrm{~A} / \mathrm{C}$ & -0.067 & 0.006 & $1.01 E-31$ & 96,598 & TG \\
\hline rs11045172 & $12 / 20361488$ & $P D E 3 A$ & $\mathbf{A} / \mathbf{C}$ & -0.029 & 0.007 & $2.83 E-06$ & 98,285 & HDL-C \\
\hline rs11045172 & $12 / 20361488$ & $P D E 3 A$ & $\mathrm{~A} / \mathrm{C}$ & 0.019 & 0.007 & $3.38 \mathrm{E}-03$ & 94,984 & TG \\
\hline rs12812995 & $12 / 20369141$ & $P D E 3 A$ & $\mathbf{C} / \mathbf{G}$ & 0.019 & 0.005 & $2.72 E-04$ & 99,900 & HDL-C \\
\hline rs12812995 & $12 / 20369141$ & $P D E 3 A$ & $\mathrm{C} / \mathrm{G}$ & -0.012 & 0.005 & $1.33 \mathrm{E}-02$ & 96,598 & $\mathrm{TG}$ \\
\hline rs1444636 & $12 / 20384150$ & $P D E 3 A$ & $\mathrm{C} / \mathrm{G}$ & 0.019 & 0.005 & $1.46 \mathrm{E}-04$ & 99,876 & HDL-C \\
\hline rs 1444636 & $12 / 20384150$ & $P D E 3 A$ & $\mathrm{C} / \mathrm{G}$ & -0.013 & 0.005 & $5.18 \mathrm{E}-03$ & 96,574 & $\mathrm{TG}$ \\
\hline rs10770643 & $12 / 20385101$ & $P D E 3 A$ & $\mathbf{A} / \mathbf{G}$ & -0.019 & 0.005 & $1.34 E-04$ & 99,900 & HDL-C \\
\hline rs10770643 & $12 / 20385101$ & $P D E 3 A$ & $\mathrm{~A} / \mathrm{G}$ & 0.012 & 0.005 & $7.94 \mathrm{E}-03$ & 96,598 & TG \\
\hline rs7303397 & $12 / 20385638$ & $P D E 3 A$ & $\mathbf{A} / \mathbf{G}$ & -0.019 & 0.005 & $1.65 \mathrm{E}-04$ & 99,900 & HDL-C \\
\hline rs7303397 & $12 / 20385638$ & $P D E 3 A$ & $\mathrm{~A} / \mathrm{G}$ & 0.012 & 0.005 & $1.08 \mathrm{E}-02$ & 96,598 & TG \\
\hline rs7955516 & $12 / 20389303$ & $P D E 3 A$ & $\mathrm{~A} / \mathrm{C}$ & -0.018 & 0.005 & $2.37 E-04$ & 99,890 & HDL-C \\
\hline rs7955516 & $12 / 20389303$ & $P D E 3 A$ & $\mathrm{~A} / \mathrm{C}$ & 0.011 & 0.005 & $1.42 \mathrm{E}-02$ & 96,588 & TG \\
\hline rs 2120757 & $12 / 20393267$ & $P D E 3 A$ & $\mathrm{~T} / \mathrm{C}$ & -0.016 & 0.005 & $6.60 \mathrm{E}-04$ & 99,900 & HDL-C \\
\hline rs2120757 & $12 / 20393267$ & $P D E 3 A$ & $\mathrm{~T} / \mathrm{C}$ & 0.009 & 0.005 & $3.73 \mathrm{E}-02$ & 96,598 & $\mathrm{TG}$ \\
\hline rs3943606 & $12 / 20399304$ & $P D E 3 A$ & $\mathrm{~T} / \mathrm{C}$ & 0.016 & 0.005 & $6.07 \mathrm{E}-04$ & 99,900 & HDL-C \\
\hline
\end{tabular}




\begin{tabular}{|c|c|c|c|c|c|c|c|c|}
\hline rs3943606 & $12 / 20399304$ & $P D E 3 A$ & $\mathrm{~T} / \mathrm{C}$ & -0.010 & 0.005 & $2.95 \mathrm{E}-02$ & 96,598 & TG \\
\hline rs2009084 & $12 / 20402460$ & $P D E 3 A$ & $\mathrm{~T} / \mathrm{C}$ & -0.017 & 0.005 & $5.21 \mathrm{E}-04$ & 99,900 & HDL-C \\
\hline rs 2009084 & $12 / 20402460$ & $P D E 3 A$ & $\mathrm{~T} / \mathrm{C}$ & 0.012 & 0.005 & $8.39 \mathrm{E}-03$ & 96,598 & TG \\
\hline rs11057405 & $12 / 121347850$ & CLIPI & $\mathrm{A} / \mathrm{G}$ & -0.026 & 0.010 & $3.24 \mathrm{E}-03$ & 99,179 & HDL-C \\
\hline rs2454722 & $12 / 121737171$ & GPR109A & $\mathbf{A} / \mathbf{G}$ & -0.031 & 0.006 & 3.19E-07 & 99,179 & HDL-C \\
\hline rs 2454722 & $12 / 121737171$ & GPR109A & $\mathrm{A} / \mathrm{G}$ & -0.017 & 0.007 & $8.58 \mathrm{E}-03$ & 99,463 & $\mathrm{TC}$ \\
\hline rs 2454722 & $12 / 121737171$ & GPR109A & $\mathrm{A} / \mathrm{G}$ & 0.018 & 0.006 & $5.30 \mathrm{E}-03$ & 95,877 & TG \\
\hline rs601339 & $12 / 121740696$ & GPR109A & $\mathbf{A} / \mathbf{G}$ & -0.031 & 0.006 & 5.59E-07 & 99,179 & HDL-C \\
\hline rs601339 & $12 / 121740696$ & GPR109A & $\mathrm{A} / \mathrm{G}$ & -0.018 & 0.007 & $5.00 \mathrm{E}-03$ & 99,463 & $\mathrm{TC}$ \\
\hline rs601339 & $12 / 121740696$ & GPR109A & $\mathrm{A} / \mathrm{G}$ & 0.017 & 0.006 & $8.26 \mathrm{E}-03$ & 95,877 & TG \\
\hline rs4759361 & $12 / 121744233$ & GPR109A & $\mathbf{A} / \mathbf{T}$ & 0.030 & 0.006 & $1.00 \mathrm{E}-06$ & 99,179 & HDL-C \\
\hline rs4759361 & $12 / 121744233$ & GPR109A & $\mathrm{A} / \mathrm{T}$ & 0.019 & 0.007 & $3.95 \mathrm{E}-03$ & 99,463 & $\mathrm{TC}$ \\
\hline rs4759361 & $12 / 121744233$ & GPR109A & $\mathrm{A} / \mathrm{T}$ & -0.015 & 0.006 & $2.43 \mathrm{E}-02$ & 95,877 & TG \\
\hline rs509548 & $12 / 121747808$ & GPR109A & $\mathbf{A} / \mathbf{T}$ & 0.031 & 0.006 & 3.40E-07 & 99,179 & HDL-C \\
\hline rs509548 & $12 / 121747808$ & GPR109A & $\mathrm{A} / \mathrm{T}$ & 0.017 & 0.007 & $8.13 \mathrm{E}-03$ & 99,463 & $\mathrm{TC}$ \\
\hline rs509548 & $12 / 121747808$ & GPR109A & $\mathrm{A} / \mathrm{T}$ & -0.018 & 0.006 & $4.72 \mathrm{E}-03$ & 95,877 & TG \\
\hline rs6488898 & $12 / 122769785$ & ATP6V0A2 & $\mathrm{A} / \mathrm{G}$ & 0.024 & 0.010 & $5.10 \mathrm{E}-03$ & 95,716 & HDL-C \\
\hline rs11057354 & $12 / 122833591$ & DNAH1O & $\mathrm{A} / \mathrm{G}$ & -0.024 & 0.008 & $2.37 \mathrm{E}-03$ & 99,894 & HDL-C \\
\hline rs11057354 & $12 / 122833591$ & DNAH10 & $\mathrm{A} / \mathrm{G}$ & 0.016 & 0.008 & $3.74 \mathrm{E}-02$ & 96,592 & TG \\
\hline rs12317176 & $12 / 122970671$ & DNAH10 & $\mathbf{T} / \mathbf{C}$ & -0.032 & 0.005 & $9.29 \mathrm{E}-10$ & 99,900 & HDL-C \\
\hline rs 12317176 & $12 / 122970671$ & DNAH10 & $\mathrm{T} / \mathrm{C}$ & 0.018 & 0.005 & $1.44 \mathrm{E}-03$ & 95,454 & LDL-C \\
\hline rs 12317176 & $12 / 122970671$ & DNAH10 & $\mathrm{T} / \mathrm{C}$ & 0.015 & 0.005 & $8.26 \mathrm{E}-03$ & 100,184 & $\mathrm{TC}$ \\
\hline rs12317176 & $12 / 122970671$ & DNAH10 & $\mathbf{T} / \mathbf{C}$ & 0.026 & 0.005 & 3.13E-07 & 96,598 & TG \\
\hline rs7301953 & $12 / 122971824$ & DNAH10 & $\mathbf{A} / \mathbf{G}$ & 0.030 & 0.005 & $1.50 \mathrm{E}-08$ & 99,900 & HDL-C \\
\hline rs7301953 & $12 / 122971824$ & DNAH10 & $\mathrm{A} / \mathrm{G}$ & -0.018 & 0.006 & $1.19 \mathrm{E}-03$ & 95,454 & LDL-C \\
\hline rs 7301953 & $12 / 122971824$ & DNAH10 & $\mathrm{A} / \mathrm{G}$ & -0.017 & 0.005 & $4.10 \mathrm{E}-03$ & 100,184 & $\mathrm{TC}$ \\
\hline rs7301953 & $12 / 122971824$ & DNAH10 & $\mathbf{A} / \mathbf{G}$ & -0.026 & 0.005 & 6.12E-07 & 96,598 & TG \\
\hline rs10846579 & $12 / 122973356$ & DNAH10 & $\mathbf{T} / \mathbf{C}$ & 0.032 & 0.005 & 9.57E-10 & 99,900 & HDL-C \\
\hline rs 10846579 & $12 / 122973356$ & DNAH10 & $\mathrm{T} / \mathrm{C}$ & -0.018 & 0.005 & $1.15 \mathrm{E}-03$ & 95,454 & LDL-C \\
\hline rs10846579 & $12 / 122973356$ & DNAH10 & $\mathrm{T} / \mathrm{C}$ & -0.016 & 0.005 & $7.01 \mathrm{E}-03$ & 100,184 & $\mathrm{TC}$ \\
\hline rs10846579 & $12 / 122973356$ & DNAH10 & $\mathbf{T} / \mathbf{C}$ & -0.026 & 0.005 & 2.48E-07 & 96,598 & TG \\
\hline rs11057394 & $12 / 122973629$ & DNAH10 & $\mathbf{T} / \mathbf{C}$ & -0.031 & 0.005 & $1.54 \mathrm{E}-09$ & 99,900 & HDL-C \\
\hline rs11057394 & $12 / 122973629$ & DNAH10 & $\mathrm{T} / \mathrm{C}$ & 0.018 & 0.005 & $8.30 \mathrm{E}-04$ & 95,454 & LDL-C \\
\hline rs11057394 & $12 / 122973629$ & DNAH10 & $\mathrm{T} / \mathrm{C}$ & 0.016 & 0.005 & $4.85 \mathrm{E}-03$ & 100,184 & $\mathrm{TC}$ \\
\hline rs11057394 & $12 / 122973629$ & DNAH10 & $\mathbf{T} / \mathbf{C}$ & 0.026 & 0.005 & 1.79E-07 & 96,598 & TG \\
\hline rs12809125 & $12 / 122973944$ & DNAH10 & A/G & -0.032 & 0.005 & 8.16E-10 & 99,900 & HDL-C \\
\hline rs12809125 & $12 / 122973944$ & DNAH10 & $\mathrm{A} / \mathrm{G}$ & 0.018 & 0.005 & $1.06 \mathrm{E}-03$ & 95,454 & LDL-C \\
\hline rs12809125 & $12 / 122973944$ & DNAH10 & $\mathrm{A} / \mathrm{G}$ & 0.016 & 0.005 & $6.00 \mathrm{E}-03$ & 100,184 & $\mathrm{TC}$ \\
\hline rs12809125 & $12 / 122973944$ & DNAH10 & $\mathbf{A} / \mathbf{G}$ & 0.026 & 0.005 & $1.74 \mathrm{E}-07$ & 96,598 & TG \\
\hline rs7133378 & $12 / 122975455$ & DNAH10 & $\mathbf{A} / \mathbf{G}$ & 0.031 & 0.005 & 3.72E-09 & 99,900 & HDL-C \\
\hline rs 7133378 & $12 / 122975455$ & DNAH10 & $\mathrm{A} / \mathrm{G}$ & -0.018 & 0.006 & $1.91 \mathrm{E}-03$ & 95,454 & LDL-C \\
\hline rs7133378 & $12 / 122975455$ & DNAH10 & $\mathrm{A} / \mathrm{G}$ & -0.015 & 0.006 & $1.14 \mathrm{E}-02$ & 100,184 & $\mathrm{TC}$ \\
\hline rs7133378 & $12 / 122975455$ & DNAH10 & A/G & -0.023 & 0.005 & $5.49 \mathrm{E}-06$ & 96,598 & TG \\
\hline rs9971695 & $12 / 122979444$ & DNAH10 & $\mathbf{A} / \mathbf{G}$ & 0.032 & 0.005 & $6.73 E-10$ & 99,900 & HDL-C \\
\hline rs9971695 & $12 / 122979444$ & DNAH10 & $\mathrm{A} / \mathrm{G}$ & -0.018 & 0.005 & $1.06 \mathrm{E}-03$ & 95,454 & LDL-C \\
\hline rs9971695 & $12 / 122979444$ & DNAHIO & $\mathrm{A} / \mathrm{G}$ & -0.016 & 0.005 & $6.26 \mathrm{E}-03$ & 100,184 & $\mathrm{TC}$ \\
\hline rs9971695 & $12 / 122979444$ & DNAH10 & $\mathbf{A} / \mathbf{G}$ & -0.026 & 0.005 & 1.61E-07 & 96,598 & TG \\
\hline rs3802999 & $12 / 122980051$ & DNAH10 & $\mathbf{T} / \mathbf{C}$ & 0.032 & 0.005 & $5.97 E-10$ & 99,900 & HDL-C \\
\hline rs3802999 & $12 / 122980051$ & DNAH10 & $\mathrm{T} / \mathrm{C}$ & -0.018 & 0.005 & $1.39 \mathrm{E}-03$ & 95,454 & LDL-C \\
\hline rs3802999 & $12 / 122980051$ & DNAH10 & $\mathrm{T} / \mathrm{C}$ & -0.015 & 0.005 & $8.26 \mathrm{E}-03$ & 100,184 & $\mathrm{TC}$ \\
\hline rs3802999 & $12 / 122980051$ & DNAH10 & $\mathbf{T} / \mathbf{C}$ & -0.026 & 0.005 & 1.77E-07 & 96,598 & TG \\
\hline rs4930721 & $12 / 122983842$ & DNAH10 & $\mathbf{T} / \mathbf{C}$ & 0.030 & 0.005 & 2.07E-08 & 99,900 & HDL-C \\
\hline rs4930721 & $12 / 122983842$ & DNAH10 & $\mathrm{T} / \mathrm{C}$ & -0.018 & 0.006 & $8.92 \mathrm{E}-04$ & 95,454 & LDL-C \\
\hline rs4930721 & $12 / 122983842$ & DNAHIO & $\mathrm{T} / \mathrm{C}$ & -0.018 & 0.005 & $2.25 \mathrm{E}-03$ & 100,184 & $\mathrm{TC}$ \\
\hline
\end{tabular}




\begin{tabular}{|c|c|c|c|c|c|c|c|c|}
\hline rs4930721 & $12 / 122983842$ & DNAH10 & $\mathrm{T} / \mathrm{C}$ & -0.027 & 0.005 & 1.74E-07 & 96,598 & TG \\
\hline rs12298484 & $12 / 122984627$ & DNAH10 & $\mathbf{T} / \mathbf{C}$ & 0.031 & 0.005 & 1.32E-09 & 99,846 & HDL-C \\
\hline rs12298484 & $12 / 122984627$ & DNAH10 & $\mathrm{T} / \mathrm{C}$ & -0.018 & 0.005 & $1.22 \mathrm{E}-03$ & 95,400 & LDL-C \\
\hline rs12298484 & $12 / 122984627$ & DNAH1O & $\mathrm{T} / \mathrm{C}$ & -0.016 & 0.005 & $5.48 \mathrm{E}-03$ & 100,130 & $\mathrm{TC}$ \\
\hline rs12298484 & $12 / 122984627$ & DNAH10 & $\mathbf{T} / \mathbf{C}$ & -0.027 & 0.005 & $7.52 E-08$ & 96,544 & TG \\
\hline rs11057396 & $12 / 122985015$ & DNAH10 & $\mathrm{A} / \mathrm{C}$ & -0.020 & 0.009 & 7.07E-03 & 36,612 & HDL-C \\
\hline rs11057396 & $12 / 122985015$ & DNAHIO & $\mathrm{A} / \mathrm{C}$ & 0.018 & 0.008 & $3.59 \mathrm{E}-02$ & 33,198 & TG \\
\hline rs11057397 & $12 / 122985681$ & DNAH10 & $\mathbf{T} / \mathrm{C}$ & 0.031 & 0.005 & $7.47 \mathrm{E}-10$ & $\mathbf{9 9 , 9 0 0}$ & HDL-C \\
\hline rs11057397 & $12 / 122985681$ & DNAH10 & $\mathrm{T} / \mathrm{C}$ & -0.018 & 0.005 & $8.44 \mathrm{E}-04$ & 95,454 & LDL-C \\
\hline rs11057397 & $12 / 122985681$ & DNAH10 & $\mathrm{T} / \mathrm{C}$ & -0.016 & 0.005 & $4.11 \mathrm{E}-03$ & 100,184 & $\mathrm{TC}$ \\
\hline rs11057397 & $12 / 122985681$ & DNAH10 & $\mathbf{T} / \mathrm{C}$ & -0.027 & 0.005 & $3.79 \mathrm{E}-08$ & 96,598 & TG \\
\hline rs9863 & $12 / 122987406$ & CСDC92 & $\mathbf{T} / \mathbf{C}$ & -0.025 & 0.005 & $1.35 \mathrm{E}-06$ & 99,900 & HDL-C \\
\hline rs9863 & $12 / 122987406$ & CCDC92 & $\mathrm{T} / \mathrm{C}$ & 0.017 & 0.006 & $1.57 \mathrm{E}-03$ & 95,454 & LDL-C \\
\hline rs9863 & $12 / 122987406$ & $C C D C 92$ & $\mathrm{~T} / \mathrm{C}$ & 0.016 & 0.006 & $4.01 \mathrm{E}-03$ & 100,184 & $\mathrm{TC}$ \\
\hline rs 9863 & $12 / 122987406$ & CCDC92 & $\mathbf{T} / \mathrm{C}$ & 0.023 & 0.005 & 2.94E-06 & 96,598 & TG \\
\hline rs4930723 & $12 / 122989553$ & CCDC92 & $\mathbf{C} / \mathbf{G}$ & 0.032 & 0.005 & $5.02 \mathrm{E}-10$ & 99,900 & HDL-C \\
\hline rs4930723 & $12 / 122989553$ & CCDC92 & $\mathrm{C} / \mathrm{G}$ & -0.018 & 0.005 & $8.32 \mathrm{E}-04$ & 95,454 & LDL-C \\
\hline rs4930723 & $12 / 122989553$ & $C C D C 92$ & $\mathrm{C} / \mathrm{G}$ & -0.016 & 0.005 & $5.14 \mathrm{E}-03$ & 100,184 & $\mathrm{TC}$ \\
\hline rs4930723 & $12 / 122989553$ & CCDC92 & $\mathrm{C} / \mathrm{G}$ & -0.027 & 0.005 & $7.24 \mathrm{E}-08$ & 96,598 & TG \\
\hline rs11057401 & $12 / 122993259$ & CCDC92 & $\mathbf{A} / \mathbf{T}$ & 0.030 & 0.005 & 2.19E-08 & 96,797 & HDL-C \\
\hline rs11057401 & $12 / 122993259$ & CCDC92 & $\mathrm{A} / \mathrm{T}$ & -0.018 & 0.006 & $1.20 \mathrm{E}-03$ & 92,393 & LDL-C \\
\hline rs11057401 & $12 / 122993259$ & CCDC92 & $\mathrm{A} / \mathrm{T}$ & -0.017 & 0.005 & $2.75 \mathrm{E}-03$ & 97,032 & $\mathrm{TC}$ \\
\hline rs11057401 & $12 / 122993259$ & ССDC92 & $\mathbf{A} / \mathbf{T}$ & -0.027 & 0.005 & 1.53E-07 & 93,446 & TG \\
\hline rs4930726 & $12 / 122994284$ & CCDC92 & $\mathbf{T} / \mathbf{C}$ & -0.032 & 0.005 & $6.15 E-10$ & $\mathbf{9 9 , 9 0 0}$ & HDL-C \\
\hline rs4930726 & $12 / 122994284$ & CCDC92 & $\mathrm{T} / \mathrm{C}$ & 0.018 & 0.005 & $9.30 \mathrm{E}-04$ & 95,454 & LDL-C \\
\hline rs4930726 & $12 / 122994284$ & $C C D C 92$ & $\mathrm{~T} / \mathrm{C}$ & 0.016 & 0.005 & $5.49 \mathrm{E}-03$ & 100,184 & $\mathrm{TC}$ \\
\hline rs4930726 & $12 / 122994284$ & CCDC92 & $\mathrm{T} / \mathrm{C}$ & 0.027 & 0.005 & 8.13E-08 & 96,598 & TG \\
\hline rs2178663 & $12 / 122999858$ & CCDC92 & $\mathbf{T} / \mathrm{C}$ & 0.030 & 0.005 & $1.90 \mathrm{E}-08$ & 96,908 & HDL-C \\
\hline rs 2178663 & $12 / 122999858$ & CCDC92 & $\mathrm{T} / \mathrm{C}$ & -0.018 & 0.006 & $1.35 \mathrm{E}-03$ & 92,503 & LDL-C \\
\hline rs2178663 & $12 / 122999858$ & $C C D C 92$ & $\mathrm{~T} / \mathrm{C}$ & -0.017 & 0.005 & $3.27 \mathrm{E}-03$ & 97,148 & $\mathrm{TC}$ \\
\hline rs2178663 & $12 / 122999858$ & CCDC92 & $\mathrm{T} / \mathrm{C}$ & -0.027 & 0.005 & $1.59 \mathrm{E}-07$ & 93,562 & TG \\
\hline rs4405410 & $12 / 123001741$ & CCDC92 & $\mathbf{A} / \mathbf{T}$ & 0.032 & 0.005 & $6.28 \mathrm{E}-10$ & 99,900 & HDL-C \\
\hline rs 4405410 & $12 / 123001741$ & $C C D C 92$ & $\mathrm{~A} / \mathrm{T}$ & -0.018 & 0.005 & $9.06 \mathrm{E}-04$ & 95,454 & LDL-C \\
\hline rs4405410 & $12 / 123001741$ & $C C D C 92$ & $\mathrm{~A} / \mathrm{T}$ & -0.016 & 0.005 & $5.20 \mathrm{E}-03$ & 100,184 & $\mathrm{TC}$ \\
\hline rs4405410 & $12 / 123001741$ & CCDC92 & $\mathbf{A} / \mathbf{T}$ & -0.027 & 0.005 & $7.87 \mathrm{E}-08$ & 96,598 & TG \\
\hline rs7961449 & $12 / 123003168$ & CCDC92 & $\mathbf{A} / \mathbf{T}$ & 0.032 & 0.005 & $6.29 \mathrm{E}-10$ & 99,900 & HDL-C \\
\hline rs 7961449 & $12 / 123003168$ & CCDC92 & $\mathrm{A} / \mathrm{T}$ & -0.018 & 0.005 & $9.64 \mathrm{E}-04$ & 95,454 & LDL-C \\
\hline rs 7961449 & $12 / 123003168$ & CCDC92 & $\mathrm{A} / \mathrm{T}$ & -0.016 & 0.005 & $5.59 \mathrm{E}-03$ & 100,184 & $\mathrm{TC}$ \\
\hline rs7961449 & $12 / 123003168$ & ССDC92 & $\mathbf{A} / \mathbf{T}$ & -0.027 & 0.005 & $6.45 E-08$ & 96,598 & TG \\
\hline rs7964945 & $12 / 123003621$ & CCDC92 & $\mathbf{A} / \mathbf{T}$ & 0.022 & 0.007 & 4.53E-04 & 99,900 & HDL-C \\
\hline rs7964945 & $12 / 123003621$ & CCDC92 & $\mathrm{A} / \mathrm{T}$ & -0.025 & 0.008 & $8.13 \mathrm{E}-04$ & 95,454 & LDL-C \\
\hline rs7964945 & $12 / 123003621$ & $C C D C 92$ & $\mathrm{~A} / \mathrm{T}$ & -0.023 & 0.007 & $3.99 \mathrm{E}-03$ & 100,184 & $\mathrm{TC}$ \\
\hline rs 7964945 & $12 / 123003621$ & CCDC92 & $\mathrm{A} / \mathrm{T}$ & -0.022 & 0.007 & $1.89 \mathrm{E}-03$ & 96,598 & TG \\
\hline rs3867146 & $12 / 123003958$ & CCDC92 & $\mathbf{A} / \mathbf{G}$ & -0.031 & 0.008 & 1.64E-04 & 99,900 & HDL-C \\
\hline rs3867146 & $12 / 123003958$ & CCDC92 & $\mathrm{A} / \mathrm{G}$ & 0.024 & 0.008 & $3.07 \mathrm{E}-03$ & 96,598 & TG \\
\hline rs7132655 & $12 / 123004926$ & CCDC92 & $\mathbf{C} / \mathbf{G}$ & 0.032 & 0.005 & 1.03E-09 & 96,908 & HDL-C \\
\hline rs7132655 & $12 / 123004926$ & $C C D C 92$ & $\mathrm{C} / \mathrm{G}$ & -0.018 & 0.005 & $1.26 \mathrm{E}-03$ & 92,503 & LDL-C \\
\hline rs7132655 & $12 / 123004926$ & $C C D C 92$ & $\mathrm{C} / \mathrm{G}$ & -0.016 & 0.005 & $6.64 \mathrm{E}-03$ & 97,148 & $\mathrm{TC}$ \\
\hline rs7132655 & $12 / 123004926$ & CCDC92 & $\mathrm{C} / \mathrm{G}$ & -0.027 & 0.005 & 1.06E-07 & 93,562 & TG \\
\hline rs4765219 & $12 / 123006063$ & CCDC92 & $\mathbf{A} / \mathbf{C}$ & 0.033 & 0.005 & $4.36 \mathrm{E}-10$ & 99,871 & HDL-C \\
\hline rs4765219 & $12 / 123006063$ & $C C D C 92$ & $\mathrm{~A} / \mathrm{C}$ & -0.018 & 0.005 & $9.41 \mathrm{E}-04$ & 95,427 & LDL-C \\
\hline rs4765219 & $12 / 123006063$ & CCDC92 & $\mathrm{A} / \mathrm{C}$ & -0.016 & 0.005 & $6.26 \mathrm{E}-03$ & 100,151 & $\mathrm{TC}$ \\
\hline rs4765219 & $12 / 123006063$ & CCDC 92 & $\mathbf{A} / \mathbf{C}$ & -0.027 & 0.005 & 1.06E-07 & 96,565 & TG \\
\hline rs7958691 & $12 / 123006696$ & CCDC92 & $\mathbf{T} / \mathbf{G}$ & 0.032 & 0.005 & $5.59 \mathrm{E}-10$ & 99,900 & HDL-C \\
\hline
\end{tabular}




\begin{tabular}{|c|c|c|c|c|c|c|c|c|}
\hline rs7958691 & $12 / 123006696$ & CCDC92 & $\mathrm{T} / \mathrm{G}$ & -0.018 & 0.005 & $7.51 \mathrm{E}-04$ & 95,454 & LDL-C \\
\hline rs7958691 & $12 / 123006696$ & $C C D C 92$ & $\mathrm{~T} / \mathrm{G}$ & -0.016 & 0.005 & $5.45 \mathrm{E}-03$ & 100,184 & $\mathrm{TC}$ \\
\hline rs7958691 & $12 / 123006696$ & ССDC92 & $\mathbf{T} / \mathbf{G}$ & -0.027 & 0.005 & 8.78E-08 & 96,598 & TG \\
\hline rs7305864 & $12 / 123007833$ & ССDC92 & $\mathbf{C} / \mathbf{G}$ & -0.032 & 0.005 & 1.17E-09 & 99,686 & HDL-C \\
\hline rs7305864 & $12 / 123007833$ & CCDC92 & $\mathrm{C} / \mathrm{G}$ & 0.019 & 0.005 & $6.75 \mathrm{E}-04$ & 95,243 & LDL-C \\
\hline rs7305864 & $12 / 123007833$ & CCDC92 & $\mathrm{C} / \mathrm{G}$ & 0.016 & 0.005 & $4.16 \mathrm{E}-03$ & 99,970 & $\mathrm{TC}$ \\
\hline rs7305864 & $12 / 123007833$ & CCDC92 & $\mathrm{C} / \mathrm{G}$ & 0.026 & 0.005 & 1.79E-07 & 96,384 & TG \\
\hline rs6488913 & $12 / 123011522$ & CCDC92 & $\mathrm{C} / \mathrm{G}$ & 0.033 & 0.005 & $3.88 \mathrm{E}-10$ & 99,900 & HDL-C \\
\hline rs6488913 & $12 / 123011522$ & CCDC92 & $\mathrm{C} / \mathrm{G}$ & -0.018 & 0.005 & 7.62E-04 & 95,454 & LDL-C \\
\hline rs6488913 & $12 / 123011522$ & CCDC92 & $\mathrm{C} / \mathrm{G}$ & -0.016 & 0.005 & $5.61 \mathrm{E}-03$ & 100,184 & $\mathrm{TC}$ \\
\hline rs6488913 & $12 / 123011522$ & ССDC92 & $\mathrm{C} / \mathrm{G}$ & -0.027 & 0.005 & 7.33E-08 & 96,598 & TG \\
\hline rs7312404 & $12 / 123012681$ & CCDC92 & $\mathrm{A} / \mathrm{G}$ & -0.019 & 0.009 & $1.03 \mathrm{E}-02$ & 36,612 & HDL-C \\
\hline rs7312404 & $12 / 123012681$ & CCDC92 & $\mathrm{A} / \mathrm{G}$ & 0.018 & 0.008 & $3.21 \mathrm{E}-02$ & 33,198 & TG \\
\hline rs11831913 & $12 / 123012851$ & ССDC92 & $\mathbf{T} / \mathbf{C}$ & -0.031 & 0.005 & 7.79E-09 & 99,900 & HDL-C \\
\hline rs11831913 & $12 / 123012851$ & CCDC92 & $\mathrm{T} / \mathrm{C}$ & 0.019 & 0.005 & $8.65 \mathrm{E}-04$ & 95,454 & LDL-C \\
\hline rs11831913 & $12 / 123012851$ & CCDC92 & $\mathrm{T} / \mathrm{C}$ & 0.017 & 0.005 & $2.80 \mathrm{E}-03$ & 100,184 & $\mathrm{TC}$ \\
\hline rs11831913 & $12 / 123012851$ & CCDC92 & $\mathbf{T} / \mathbf{C}$ & 0.028 & 0.005 & $8.76 \mathrm{E}-08$ & 96,598 & TG \\
\hline rs6488914 & $12 / 123013794$ & CCDC92 & $\mathrm{C} / \mathrm{G}$ & 0.031 & 0.005 & 1.01E-08 & $\mathbf{9 9 , 8 7 3}$ & HDL-C \\
\hline rs6488914 & $12 / 123013794$ & CCDC92 & $\mathrm{C} / \mathrm{G}$ & -0.019 & 0.006 & 7.33E-04 & 95,427 & LDL-C \\
\hline rs6488914 & $12 / 123013794$ & CCDC92 & $\mathrm{C} / \mathrm{G}$ & -0.018 & 0.005 & $2.26 \mathrm{E}-03$ & 100,157 & $\mathrm{TC}$ \\
\hline rs6488914 & $12 / 123013794$ & ССDC92 & $\mathrm{C} / \mathrm{G}$ & -0.028 & 0.005 & 1.04E-07 & 96,571 & TG \\
\hline rs7973683 & $12 / 123015176$ & CCDC92 & $\mathbf{A} / \mathbf{C}$ & 0.032 & 0.005 & $3.46 \mathrm{E}-10$ & 99,900 & HDL-C \\
\hline rs7973683 & $12 / 123015176$ & CCDC92 & $\mathrm{A} / \mathrm{C}$ & -0.018 & 0.005 & $9.07 \mathrm{E}-04$ & 95,454 & LDL-C \\
\hline rs7973683 & $12 / 123015176$ & CCDC92 & $\mathrm{A} / \mathrm{C}$ & -0.016 & 0.005 & $6.12 \mathrm{E}-03$ & 100,184 & $\mathrm{TC}$ \\
\hline rs7973683 & $12 / 123015176$ & ССDC92 & $\mathbf{A} / \mathbf{C}$ & -0.027 & 0.005 & 5.91E-08 & 96,598 & TG \\
\hline rs4765127 & $12 / 123026120$ & ZNF664 & $\mathbf{T} / \mathbf{G}$ & 0.033 & 0.005 & 2.89E-10 & $\mathbf{9 9 , 7 8 7}$ & HDL-C \\
\hline rs4765127 & $12 / 123026120$ & ZNF664 & $\mathrm{T} / \mathrm{G}$ & -0.018 & 0.005 & $9.36 \mathrm{E}-04$ & 95,342 & LDL-C \\
\hline rs4765127 & $12 / 123026120$ & ZNF664 & $\mathrm{T} / \mathrm{G}$ & -0.016 & 0.005 & $5.07 \mathrm{E}-03$ & 100,069 & $\mathrm{TC}$ \\
\hline rs4765127 & $12 / 123026120$ & ZNF664 & $\mathbf{T} / \mathbf{G}$ & -0.028 & 0.005 & $1.80 \mathrm{E}-08$ & 96,483 & TG \\
\hline rs12311114 & $12 / 123026656$ & ZNF664 & $\mathbf{A} / \mathbf{C}$ & 0.031 & 0.005 & 8.49E-09 & 99,820 & HDL-C \\
\hline rs12311114 & $12 / 123026656$ & ZNF664 & $\mathrm{A} / \mathrm{C}$ & -0.019 & 0.006 & $8.25 \mathrm{E}-04$ & 95,377 & LDL-C \\
\hline rs12311114 & $12 / 123026656$ & ZNF664 & $\mathrm{A} / \mathrm{C}$ & -0.017 & 0.005 & $2.73 \mathrm{E}-03$ & 100,103 & $\mathrm{TC}$ \\
\hline rs12311114 & $12 / 123026656$ & ZNF664 & $\mathbf{A} / \mathbf{C}$ & -0.028 & 0.005 & $9.13 E-08$ & 96,517 & TG \\
\hline rs4765528 & $12 / 123028207$ & ZNF664 & $\mathbf{A} / \mathbf{T}$ & 0.031 & 0.005 & 5.41E-09 & 99,711 & HDL-C \\
\hline rs4765528 & $12 / 123028207$ & ZNF664 & $\mathrm{A} / \mathrm{T}$ & -0.019 & 0.005 & $6.23 \mathrm{E}-04$ & 95,266 & LDL-C \\
\hline rs4765528 & $12 / 123028207$ & ZNF664 & $\mathrm{A} / \mathrm{T}$ & -0.017 & 0.005 & $2.67 \mathrm{E}-03$ & 99,987 & $\mathrm{TC}$ \\
\hline rs4765528 & $12 / 123028207$ & ZNF664 & $\mathbf{A} / \mathbf{T}$ & -0.028 & 0.005 & 5.17E-08 & 96,401 & TG \\
\hline rs11057408 & $12 / 123030789$ & ZNF664 & $\mathbf{T} / \mathbf{G}$ & 0.033 & 0.005 & 5.93E-10 & 96,711 & HDL-C \\
\hline rs11057408 & $12 / 123030789$ & ZNF664 & $\mathrm{T} / \mathrm{G}$ & -0.020 & 0.005 & $5.21 \mathrm{E}-04$ & 92,308 & LDL-C \\
\hline rs11057408 & $12 / 123030789$ & ZNF664 & $\mathrm{T} / \mathrm{G}$ & -0.017 & 0.005 & $2.84 \mathrm{E}-03$ & 96,943 & $\mathrm{TC}$ \\
\hline rs11057408 & $12 / 123030789$ & ZNF664 & $\mathbf{T} / \mathbf{G}$ & -0.029 & 0.005 & $1.60 \mathrm{E}-08$ & 93,357 & TG \\
\hline rs7978610 & $12 / 123034525$ & ZNF664 & $\mathrm{C} / \mathrm{G}$ & 0.033 & 0.005 & $6.36 \mathrm{E}-10$ & 96,878 & HDL-C \\
\hline rs7978610 & $12 / 123034525$ & ZNF664 & $\mathrm{C} / \mathrm{G}$ & -0.019 & 0.006 & 7.33E-04 & 92,474 & LDL-C \\
\hline rs7978610 & $12 / 123034525$ & ZNF664 & $\mathrm{C} / \mathrm{G}$ & -0.017 & 0.005 & $3.49 \mathrm{E}-03$ & 97,119 & $\mathrm{TC}$ \\
\hline rs7978610 & $12 / 123034525$ & ZNF664 & $\mathrm{C} / \mathrm{G}$ & -0.029 & 0.005 & $1.43 E-08$ & 93,532 & TG \\
\hline rs952632 & $12 / 123035691$ & ZNF664 & $\mathbf{A} / \mathbf{G}$ & -0.032 & 0.005 & 8.59E-10 & 96,908 & HDL-C \\
\hline rs952632 & $12 / 123035691$ & ZNF664 & $\mathrm{A} / \mathrm{G}$ & 0.018 & 0.005 & $1.33 \mathrm{E}-03$ & 92,503 & LDL-C \\
\hline rs952632 & $12 / 123035691$ & ZNF664 & $\mathrm{A} / \mathrm{G}$ & 0.016 & 0.005 & $6.41 \mathrm{E}-03$ & 97,148 & $\mathrm{TC}$ \\
\hline rs952632 & $12 / 123035691$ & ZNF664 & $\mathbf{A} / \mathbf{G}$ & 0.028 & 0.005 & $3.34 \mathrm{E}-08$ & 93,562 & TG \\
\hline rs11837287 & $12 / 123036012$ & ZNF664 & $\mathbf{T} / \mathbf{C}$ & 0.032 & 0.005 & $1.25 \mathrm{E}-09$ & 96,908 & HDL-C \\
\hline rs11837287 & $12 / 123036012$ & ZNF664 & $\mathrm{T} / \mathrm{C}$ & -0.019 & 0.006 & $8.90 \mathrm{E}-04$ & 92,503 & LDL-C \\
\hline rs11837287 & $12 / 123036012$ & ZNF664 & $\mathrm{T} / \mathrm{C}$ & -0.017 & 0.005 & $4.90 \mathrm{E}-03$ & 97,148 & $\mathrm{TC}$ \\
\hline rs11837287 & $12 / 123036012$ & ZNF664 & $\mathbf{T} / \mathbf{C}$ & -0.028 & 0.005 & $4.46 \mathrm{E}-08$ & 93,562 & TG \\
\hline
\end{tabular}




\begin{tabular}{|c|c|c|c|c|c|c|c|c|}
\hline rs7311969 & $12 / 123036286$ & ZNF664 & $\mathbf{T} / \mathbf{C}$ & -0.032 & 0.005 & 8.47E-10 & 99,796 & HDL-C \\
\hline rs 7311969 & $12 / 123036286$ & ZNF664 & $\mathrm{T} / \mathrm{C}$ & 0.019 & 0.005 & $8.63 \mathrm{E}-04$ & 95,350 & LDL-C \\
\hline rs7311969 & $12 / 123036286$ & ZNF664 & $\mathrm{T} / \mathrm{C}$ & 0.016 & 0.005 & 4.63E-03 & 100,076 & $\mathrm{TC}$ \\
\hline rs7311969 & $12 / 123036286$ & ZNF664 & $\mathbf{T} / \mathbf{C}$ & 0.028 & 0.005 & 3.12E-08 & 96,490 & TG \\
\hline rs7307277 & $12 / 123041109$ & ZNF664 & $\mathbf{A} / \mathbf{G}$ & -0.032 & 0.005 & 1.09E-09 & 99,900 & HDL-C \\
\hline rs 7307277 & $12 / 123041109$ & ZNF664 & $\mathrm{A} / \mathrm{G}$ & 0.019 & 0.005 & $7.43 \mathrm{E}-04$ & 95,454 & LDL-C \\
\hline rs 7307277 & $12 / 123041109$ & ZNF664 & $\mathrm{A} / \mathrm{G}$ & 0.016 & 0.005 & 4.40E-03 & 100,184 & $\mathrm{TC}$ \\
\hline rs7307277 & $12 / 123041109$ & ZNF664 & $\mathbf{A} / \mathbf{G}$ & 0.027 & 0.005 & 4.57E- 08 & 96,598 & TG \\
\hline rs 7311233 & $12 / 123041893$ & ZNF664 & $\mathbf{A} / \mathbf{G}$ & -0.030 & 0.005 & 2.17E-08 & 99,874 & HDL-C \\
\hline rs 7311233 & $12 / 123041893$ & ZNF664 & $\mathrm{A} / \mathrm{G}$ & 0.018 & 0.005 & 8.64E-04 & 95,428 & LDL-C \\
\hline rs 7311233 & $12 / 123041893$ & ZNF664 & $\mathrm{A} / \mathrm{G}$ & 0.018 & 0.005 & $2.24 \mathrm{E}-03$ & 100,157 & $\mathrm{TC}$ \\
\hline rs7311233 & $12 / 123041893$ & ZNF664 & $\mathbf{A} / \mathbf{G}$ & 0.028 & 0.005 & $7.26 \mathrm{E}-08$ & 96,571 & TG \\
\hline rs12809473 & $12 / 123042552$ & ZNF664 & $\mathbf{A} / \mathbf{T}$ & 0.032 & 0.005 & 8.55E-10 & 99,900 & HDL-C \\
\hline rs 12809473 & $12 / 123042552$ & ZNF664 & $\mathrm{A} / \mathrm{T}$ & -0.019 & 0.005 & $7.89 \mathrm{E}-04$ & 95,454 & LDL-C \\
\hline rs 12809473 & $12 / 123042552$ & ZNF664 & $\mathrm{A} / \mathrm{T}$ & -0.016 & 0.005 & 4.69E-03 & 100,184 & $\mathrm{TC}$ \\
\hline rs12809473 & $12 / 123042552$ & ZNF664 & $\mathbf{A} / \mathbf{T}$ & -0.028 & 0.005 & $3.56 \mathrm{E}-08$ & 96,598 & TG \\
\hline rs12827409 & $12 / 123042659$ & ZNF664 & $\mathbf{C} / \mathbf{G}$ & -0.032 & 0.005 & $7.28 \mathrm{E}-10$ & 99,900 & HDL-C \\
\hline rs12827409 & $12 / 123042659$ & ZNF664 & $\mathrm{C} / \mathrm{G}$ & 0.019 & 0.005 & $9.16 \mathrm{E}-04$ & 95,454 & LDL-C \\
\hline rs12827409 & $12 / 123042659$ & ZNF664 & $\mathrm{C} / \mathrm{G}$ & 0.016 & 0.005 & $5.17 \mathrm{E}-03$ & 100,184 & $\mathrm{TC}$ \\
\hline rs12827409 & $12 / 123042659$ & ZNF664 & $\mathrm{C} / \mathrm{G}$ & 0.028 & 0.005 & $3.22 \mathrm{E}-08$ & 96,598 & TG \\
\hline rs7135314 & $12 / 123044519$ & ZNF664 & $\mathbf{A} / \mathbf{T}$ & -0.032 & 0.005 & $1.06 \mathrm{E}-09$ & 99,900 & HDL-C \\
\hline rs7135314 & $12 / 123044519$ & ZNF664 & $\mathrm{A} / \mathrm{T}$ & 0.019 & 0.005 & $5.90 \mathrm{E}-04$ & 95,454 & LDL-C \\
\hline rs7135314 & $12 / 123044519$ & ZNF664 & $\mathrm{A} / \mathrm{T}$ & 0.017 & 0.005 & $3.44 \mathrm{E}-03$ & 100,184 & $\mathrm{TC}$ \\
\hline rs7135314 & $12 / 123044519$ & ZNF664 & $\mathbf{A} / \mathbf{T}$ & 0.028 & 0.005 & 3.27E-08 & 96,598 & TG \\
\hline rs4765148 & $12 / 123044590$ & ZNF664 & $\mathbf{T} / \mathbf{G}$ & 0.031 & 0.005 & $1.54 \mathrm{E}-08$ & $\mathbf{9 9 , 8 5 7}$ & HDL-C \\
\hline rs4765148 & $12 / 123044590$ & ZNF664 & $\mathrm{T} / \mathrm{G}$ & -0.019 & 0.006 & $6.18 \mathrm{E}-04$ & 95,412 & LDL-C \\
\hline rs 4765148 & $12 / 123044590$ & ZNF664 & $\mathrm{T} / \mathrm{G}$ & -0.018 & 0.005 & $1.49 \mathrm{E}-03$ & 100,140 & $\mathrm{TC}$ \\
\hline rs4765148 & $12 / 123044590$ & ZNF664 & $\mathbf{T} / \mathbf{G}$ & -0.029 & 0.005 & $3.41 \mathrm{E}-08$ & 96,554 & TG \\
\hline rs11057409 & $12 / 123045284$ & ZNF664 & $\mathbf{T} / \mathbf{C}$ & 0.032 & 0.005 & 8.24E-10 & $\mathbf{9 9 , 8 5 1}$ & HDL-C \\
\hline rs11057409 & $12 / 123045284$ & ZNF664 & $\mathrm{T} / \mathrm{C}$ & -0.019 & 0.005 & $7.96 \mathrm{E}-04$ & 95,405 & LDL-C \\
\hline rs11057409 & $12 / 123045284$ & ZNF664 & $\mathrm{T} / \mathrm{C}$ & -0.017 & 0.005 & $3.92 \mathrm{E}-03$ & 100,134 & $\mathrm{TC}$ \\
\hline rs11057409 & $12 / 123045284$ & ZNF664 & $\mathbf{T} / \mathbf{C}$ & -0.028 & 0.005 & 2.23E- 08 & 96,548 & TG \\
\hline rs7975482 & $12 / 123047643$ & ZNF664 & $\mathbf{A} / \mathbf{G}$ & -0.032 & 0.005 & $1.04 \mathrm{E}-09$ & 99,875 & HDL-C \\
\hline rs7975482 & $12 / 123047643$ & ZNF664 & $\mathrm{A} / \mathrm{G}$ & 0.019 & 0.005 & $9.15 \mathrm{E}-04$ & 95,429 & LDL-C \\
\hline rs7975482 & $12 / 123047643$ & ZNF664 & $\mathrm{A} / \mathrm{G}$ & 0.017 & 0.005 & $4.15 \mathrm{E}-03$ & 100,158 & $\mathrm{TC}$ \\
\hline rs7975482 & $12 / 123047643$ & ZNF664 & A/G & 0.028 & 0.005 & $2.42 \mathrm{E}-08$ & 96,572 & TG \\
\hline rs2130382 & $12 / 123050596$ & ZNF664 & $\mathrm{C} / \mathrm{G}$ & -0.031 & 0.005 & 2.05E-08 & 99,900 & HDL-C \\
\hline rs 2130382 & $12 / 123050596$ & ZNF664 & $\mathrm{C} / \mathrm{G}$ & 0.020 & 0.006 & $5.36 \mathrm{E}-04$ & 95,454 & LDL-C \\
\hline rs2130382 & $12 / 123050596$ & ZNF664 & $\mathrm{C} / \mathrm{G}$ & 0.018 & 0.005 & $1.62 \mathrm{E}-03$ & 100,184 & $\mathrm{TC}$ \\
\hline rs2130382 & $12 / 123050596$ & ZNF664 & $\mathrm{C} / \mathrm{G}$ & 0.028 & 0.005 & $5.25 \mathrm{E}-08$ & 96,598 & TG \\
\hline rs12310367 & $12 / 123052631$ & ZNF664 & $\mathbf{A} / \mathbf{G}$ & -0.033 & 0.005 & 3.12E-10 & 99,900 & HDL-C \\
\hline rs 12310367 & $12 / 123052631$ & ZNF664 & $\mathrm{A} / \mathrm{G}$ & 0.019 & 0.006 & 7.37E-04 & 95,454 & LDL-C \\
\hline rs 12310367 & $12 / 123052631$ & ZNF664 & $\mathrm{A} / \mathrm{G}$ & 0.016 & 0.006 & $4.29 \mathrm{E}-03$ & 100,184 & $\mathrm{TC}$ \\
\hline rs12310367 & $12 / 123052631$ & ZNF664 & $\mathbf{A} / \mathbf{G}$ & 0.028 & 0.005 & $1.21 \mathrm{E}-08$ & 96,598 & TG \\
\hline rs11057412 & $12 / 123055095$ & ZNF664 & $\mathrm{C} / \mathrm{G}$ & 0.032 & 0.005 & 4.98E-10 & 99,900 & HDL-C \\
\hline rs11057412 & $12 / 123055095$ & ZNF664 & $\mathrm{C} / \mathrm{G}$ & -0.018 & 0.006 & $1.02 \mathrm{E}-03$ & 95,454 & LDL-C \\
\hline rs11057412 & $12 / 123055095$ & ZNF664 & $\mathrm{C} / \mathrm{G}$ & -0.016 & 0.006 & $5.81 \mathrm{E}-03$ & 100,184 & $\mathrm{TC}$ \\
\hline rs11057412 & $12 / 123055095$ & ZNF664 & $\mathrm{C} / \mathrm{G}$ & -0.028 & 0.005 & 2.32E-08 & 96,598 & TG \\
\hline rs1187415 & $12 / 123057482$ & ZNF664 & $\mathrm{C} / \mathrm{G}$ & -0.032 & 0.005 & 1.33E-09 & 96,870 & HDL-C \\
\hline rs 1187415 & $12 / 123057482$ & ZNF664 & $\mathrm{C} / \mathrm{G}$ & 0.018 & 0.006 & $1.33 \mathrm{E}-03$ & 92,468 & LDL-C \\
\hline rs1187415 & $12 / 123057482$ & ZNF664 & $\mathrm{C} / \mathrm{G}$ & 0.016 & 0.006 & $4.66 \mathrm{E}-03$ & 97,109 & $\mathrm{TC}$ \\
\hline rs1187415 & $12 / 123057482$ & ZNF664 & $\mathrm{C} / \mathrm{G}$ & 0.028 & 0.005 & $1.90 \mathrm{E}-08$ & 93,523 & TG \\
\hline rs12303671 & $12 / 123058563$ & ZNF664 & $\mathbf{T} / \mathbf{G}$ & -0.032 & 0.005 & $1.60 \mathrm{E}-09$ & 96,908 & HDL-C \\
\hline rs12303671 & $12 / 123058563$ & ZNF664 & $\mathrm{T} / \mathrm{G}$ & 0.018 & 0.006 & $1.40 \mathrm{E}-03$ & 92,503 & LDL-C \\
\hline
\end{tabular}




\begin{tabular}{|c|c|c|c|c|c|c|c|c|}
\hline rs12303671 & $12 / 123058563$ & ZNF664 & $\mathrm{T} / \mathrm{G}$ & 0.016 & 0.006 & $5.72 \mathrm{E}-03$ & 97,148 & $\mathrm{TC}$ \\
\hline rs12303671 & $12 / 123058563$ & ZNF664 & $\mathbf{T} / \mathbf{G}$ & 0.028 & 0.005 & $3.62 \mathrm{E}-08$ & 93,562 & TG \\
\hline rs 7307053 & $12 / 123060493$ & ZNF664 & $\mathbf{T} / \mathbf{C}$ & 0.030 & 0.005 & 5.53E-08 & 96,899 & HDL-C \\
\hline rs 7307053 & $12 / 123060493$ & ZNF664 & $\mathrm{T} / \mathrm{C}$ & -0.018 & 0.006 & $1.94 \mathrm{E}-03$ & 92,494 & LDL-C \\
\hline rs 7307053 & $12 / 123060493$ & ZNF664 & $\mathrm{T} / \mathrm{C}$ & -0.017 & 0.006 & $3.60 \mathrm{E}-03$ & 97,138 & $\mathrm{TC}$ \\
\hline rs 7307053 & $12 / 123060493$ & ZNF664 & $\mathbf{T} / \mathbf{C}$ & -0.027 & 0.005 & 1.01E-07 & 93,552 & TG \\
\hline rs12824567 & $12 / 123061156$ & ZNF664 & $\mathrm{C} / \mathrm{G}$ & 0.030 & 0.005 & $1.47 E-08$ & 99,900 & HDL-C \\
\hline rs 12824567 & $12 / 123061156$ & ZNF664 & $\mathrm{C} / \mathrm{G}$ & -0.019 & 0.006 & $6.70 \mathrm{E}-04$ & 95,454 & LDL-C \\
\hline rs12824567 & $12 / 123061156$ & ZNF664 & $\mathrm{C} / \mathrm{G}$ & -0.018 & 0.006 & $2.07 \mathrm{E}-03$ & 100,184 & $\mathrm{TC}$ \\
\hline rs12824567 & $12 / 123061156$ & ZNF664 & $\mathrm{C} / \mathrm{G}$ & -0.028 & 0.005 & $3.97 \mathrm{E}-08$ & 96,598 & TG \\
\hline rs863750 & $12 / 123071397$ & ZNF664 & $\mathbf{T} / \mathbf{C}$ & -0.032 & 0.005 & $7.79 \mathrm{E}-10$ & $\mathbf{9 9 , 8 8 9}$ & HDL-C \\
\hline rs 863750 & $12 / 123071397$ & ZNF664 & $\mathrm{T} / \mathrm{C}$ & 0.014 & 0.005 & $2.79 \mathrm{E}-03$ & 96,587 & TG \\
\hline rs10773049 & $12 / 123072584$ & ZNF664 & $\mathbf{T} / \mathbf{C}$ & -0.030 & 0.005 & 7.32E-09 & 99,900 & HDL-C \\
\hline rs10773049 & $12 / 123072584$ & ZNF664 & $\mathrm{T} / \mathrm{C}$ & -0.012 & 0.005 & $2.66 \mathrm{E}-02$ & 100,184 & $\mathrm{TC}$ \\
\hline rs10773049 & $12 / 123072584$ & ZNF664 & $\mathrm{T} / \mathrm{C}$ & 0.012 & 0.005 & $8.76 \mathrm{E}-03$ & 96,598 & TG \\
\hline rs825453 & $12 / 123074711$ & ZNF664 & $\mathbf{A} / \mathbf{T}$ & 0.031 & 0.005 & 2.87E-09 & 99,900 & HDL-C \\
\hline rs 825453 & $12 / 123074711$ & ZNF664 & $\mathrm{A} / \mathrm{T}$ & 0.011 & 0.005 & $2.72 \mathrm{E}-02$ & 100,184 & $\mathrm{TC}$ \\
\hline rs 825453 & $12 / 123074711$ & ZNF664 & $\mathrm{A} / \mathrm{T}$ & -0.013 & 0.005 & $5.15 \mathrm{E}-03$ & 96,598 & TG \\
\hline rs11057418 & $12 / 123074929$ & ZNF664 & $\mathrm{C} / \mathrm{G}$ & -0.027 & 0.007 & 5.19E-05 & 94,822 & HDL-C \\
\hline rs11057418 & $12 / 123074929$ & ZNF664 & $\mathrm{C} / \mathrm{G}$ & 0.025 & 0.006 & $1.41 \mathrm{E}-04$ & 91,518 & TG \\
\hline rs2927328 & $16 / 80067223$ & CMIP & $\mathbf{T} / \mathbf{C}$ & -0.020 & 0.005 & 2.71E-05 & 98,409 & HDL-C \\
\hline rs 2927328 & $16 / 80067223$ & CMIP & $\mathrm{T} / \mathrm{C}$ & 0.017 & 0.005 & $8.01 \mathrm{E}-03$ & 95,070 & TG \\
\hline rs1471379 & 16/80068200 & CMIP & $\mathbf{T} / \mathbf{C}$ & -0.023 & 0.005 & 4.68E-06 & 96,852 & HDL-C \\
\hline rs 1471379 & $16 / 80068200$ & CMIP & $\mathrm{T} / \mathrm{C}$ & 0.012 & 0.005 & $4.48 \mathrm{E}-02$ & 93,505 & TG \\
\hline rs1966957 & 16/80068580 & CMIP & $\mathrm{C} / \mathrm{G}$ & 0.023 & 0.005 & 2.67E-06 & 98,409 & HDL-C \\
\hline rs 1966957 & $16 / 80068580$ & CMIP & $\mathrm{C} / \mathrm{G}$ & -0.013 & 0.005 & $2.52 \mathrm{E}-02$ & 95,070 & TG \\
\hline rs2927327 & 16/80069035 & СМIP & $\mathbf{A} / \mathbf{T}$ & 0.023 & 0.005 & 3.33E-06 & 98,409 & HDL-C \\
\hline rs2927327 & $16 / 80069035$ & CMIP & $\mathrm{A} / \mathrm{T}$ & -0.012 & 0.005 & $2.85 \mathrm{E}-02$ & 95,070 & TG \\
\hline rs2966079 & 16/80069307 & CMIP & $\mathbf{T} / \mathbf{C}$ & 0.020 & 0.005 & $7.92 \mathrm{E}-06$ & 98,409 & HDL-C \\
\hline rs1471152 & $16 / 80070137$ & CMIP & $\mathbf{T} / \mathbf{G}$ & -0.023 & 0.005 & $3.15 E-06$ & 98,313 & HDL-C \\
\hline rs 1471152 & $16 / 80070137$ & CMIP & $\mathrm{T} / \mathrm{G}$ & 0.012 & 0.005 & $2.70 \mathrm{E}-02$ & 94,971 & TG \\
\hline rs2927324 & $16 / 80070322$ & CMIP & $\mathbf{T} / \mathbf{C}$ & 0.021 & 0.005 & 4.79E- 06 & 98,386 & HDL-C \\
\hline rs2927323 & $16 / 80070449$ & СMIP & $\mathbf{A} / \mathbf{G}$ & 0.022 & 0.005 & $9.20 \mathrm{E}-06$ & 98,409 & HDL-C \\
\hline rs 2927323 & $16 / 80070449$ & CMIP & $\mathrm{A} / \mathrm{G}$ & -0.017 & 0.005 & $3.33 \mathrm{E}-03$ & 95,070 & TG \\
\hline rs2927322 & 16/80072006 & CMIP & $\mathbf{A} / \mathbf{G}$ & -0.025 & 0.006 & $2.00 \mathrm{E}-06$ & 98,409 & HDL-C \\
\hline rs 2927322 & $16 / 80072006$ & CMIP & $\mathrm{A} / \mathrm{G}$ & 0.017 & 0.005 & $1.01 \mathrm{E}-02$ & 95,070 & TG \\
\hline rs2966085 & $16 / 80074135$ & СMIP & $\mathbf{A} / \mathbf{G}$ & -0.020 & 0.005 & $7.10 \mathrm{E}-06$ & 98,256 & HDL-C \\
\hline rs 2966085 & $16 / 80074135$ & CMIP & $\mathrm{A} / \mathrm{G}$ & 0.015 & 0.005 & $1.07 \mathrm{E}-02$ & 94,917 & TG \\
\hline rs2317241 & 16/80077267 & CMIP & $\mathbf{A} / \mathbf{G}$ & -0.025 & 0.005 & $1.71 \mathrm{E}-06$ & 98,409 & HDL-C \\
\hline rs2317241 & $16 / 80077267$ & CMIP & $\mathrm{A} / \mathrm{G}$ & 0.018 & 0.005 & $1.27 \mathrm{E}-03$ & 95,070 & TG \\
\hline rs12443634 & $16 / 80081775$ & СМIP & $\mathrm{A} / \mathrm{C}$ & -0.032 & 0.006 & 2.01E-09 & 98,409 & HDL-C \\
\hline rs12443634 & $16 / 80081775$ & СМIP & $\mathbf{A} / \mathbf{C}$ & 0.022 & 0.005 & 2.00E-04 & 95,070 & TG \\
\hline rs2925979 & 16/80092291 & CMIP & $\mathbf{T} / \mathbf{C}$ & -0.035 & 0.005 & 2.09E-11 & 98,409 & HDL-C \\
\hline rs2925979 & $16 / 80092291$ & CMIP & $\mathbf{T} / \mathbf{C}$ & 0.022 & 0.005 & 8.97E-05 & 95,070 & TG \\
\hline rs2966093 & 16/80096121 & CMIP & $\mathbf{A} / \mathbf{G}$ & 0.023 & 0.005 & 1.02E-05 & 98,369 & HDL-C \\
\hline rs2966093 & $16 / 80096121$ & CMIP & $\mathrm{A} / \mathrm{G}$ & -0.012 & 0.005 & $3.74 \mathrm{E}-02$ & 95,031 & TG \\
\hline rs2966094 & 16/80096138 & СМIP & $\mathbf{A} / \mathbf{C}$ & -0.025 & 0.005 & $1.63 E-06$ & 98,409 & HDL-C \\
\hline rs2966094 & $16 / 80096138$ & CMIP & $\mathrm{A} / \mathrm{C}$ & 0.014 & 0.005 & $2.12 \mathrm{E}-02$ & 95,070 & TG \\
\hline rs2927307 & 16/80101333 & CMIP & $\mathbf{A} / \mathbf{G}$ & 0.017 & 0.005 & 1.09E-04 & 98,409 & HDL-C \\
\hline rs2966095 & 16/80106959 & CMIP & $\mathbf{A} / \mathbf{G}$ & -0.022 & 0.005 & 1.79E-05 & 98,409 & HDL-C \\
\hline rs2966097 & 16/80107209 & CMIP & $\mathbf{T} / \mathbf{C}$ & 0.023 & 0.006 & $1.72 \mathrm{E}-05$ & 88,953 & HDL-C \\
\hline rs 2966097 & $16 / 80107209$ & CMIP & $\mathrm{T} / \mathrm{C}$ & -0.015 & 0.006 & $1.28 \mathrm{E}-02$ & 85,615 & TG \\
\hline rs889140 & 19/38580840 & PEPD & $\mathbf{A} / \mathbf{G}$ & 0.020 & 0.005 & $1.71 \mathrm{E}-05$ & 98,409 & HDL-C \\
\hline rs 889140 & $19 / 38580840$ & $P E P D$ & $\mathrm{~A} / \mathrm{G}$ & -0.013 & 0.005 & $1.07 \mathrm{E}-02$ & 95,070 & TG \\
\hline
\end{tabular}




\begin{tabular}{lllllllll} 
rs889139 & $\mathbf{1 9 / 3 8 5 8 1 2 0 9}$ & $\boldsymbol{P E P D}$ & $\mathbf{A} / \mathbf{G}$ & $\mathbf{- 0 . 0 2 0}$ & $\mathbf{0 . 0 0 5}$ & $\mathbf{2 . 1 9 E}-\mathbf{0 5}$ & $\mathbf{9 8 , 3 8 6}$ & HDL-C \\
rs889139 & $19 / 38581209$ & $P E P D$ & $\mathrm{~A} / \mathrm{G}$ & 0.013 & 0.005 & $1.33 \mathrm{E}-02$ & 95,047 & TG \\
\hline rs731839 & $\mathbf{1 9 / 3 8 5 9 0 9 0 5}$ & $\boldsymbol{P E P D}$ & $\mathbf{A} / \mathbf{G}$ & $\mathbf{0 . 0 2 5}$ & $\mathbf{0 . 0 0 5}$ & $\mathbf{1 . 0 9 E}-06$ & $\mathbf{9 8 , 4 0 9}$ & HDL-C \\
rs731839 & $\mathbf{1 9 / 3 8 5 9 0 9 0 5}$ & $\boldsymbol{P E P D}$ & $\mathbf{A} / \mathbf{G}$ & $\mathbf{- 0 . 0 2 0}$ & $\mathbf{0 . 0 0 5}$ & $\mathbf{2 . 1 5 E - 0 4}$ & $\mathbf{9 5 , 0 7 0}$ & TG \\
rs4805885 & $\mathbf{1 9 / 3 8 5 9 7 9 6 3}$ & $\boldsymbol{P E P D}$ & T/C & $\mathbf{- 0 . 0 2 5}$ & $\mathbf{0 . 0 0 5}$ & $\mathbf{6 . 8 1 E - 0 7}$ & $\mathbf{9 8 , 4 0 9}$ & HDL-C \\
rs4805885 & $19 / 38597963$ & $P E P D$ & $\mathrm{~T} / \mathrm{C}$ & 0.016 & 0.005 & $2.54 \mathrm{E}-03$ & 95,070 & TG \\
rs8182584 & $\mathbf{1 9 / 3 8 6 0 1 5 5 0}$ & $\boldsymbol{P E P D}$ & T/G & $\mathbf{- 0 . 0 2 5}$ & $\mathbf{0 . 0 0 5}$ & $\mathbf{3 . 1 9 E - 0 7}$ & $\mathbf{9 8 , 4 0 9}$ & HDL-C \\
rs8182584 & $19 / 38601550$ & $P E P D$ & $\mathrm{~T} / \mathrm{G}$ & 0.015 & 0.005 & $3.62 \mathrm{E}-03$ & 95,070 & TG \\
\hline
\end{tabular}

For these traits the effect size is in SD units, based on standard error-weighted metaanalysis. *Results that are statistically significant, accounting for the number of independent SNPs are highlighted in bold.

EA: Effect Allele

NEA: Non-Effect Allele

EA-Freq: Frequency of Effect Allele 
